ACCEPted For publication in the Astrophysical Journal

Preprint typeset using $\mathrm{LATEX}_{\mathrm{E}}$ style emulateapj v. 08/22/09

\title{
DYNAMICAL ORIGIN OF EXTRASOLAR PLANET ECCENTRICITY DISTRIBUTION
}

\author{
Mario Jurić ${ }^{1,2}$, Scott Tremaine ${ }^{1,2}$ \\ Accepted for publication in the Astrophysical Journal
}

\begin{abstract}
We explore the possibility that the observed eccentricity distribution of extrasolar planets arose through planet-planet interactions, after the initial stage of planet formation was complete. Our results are based on $\sim 3250$ numerical integrations of ensembles of randomly constructed planetary systems, each lasting $100 \mathrm{Myr}$. We find that for a remarkably wide range of initial conditions the eccentricity distributions of dynamically active planetary systems relax towards a common final equilibrium distribution, well described by the fitting formula $d n \propto e \exp \left[-\frac{1}{2}(e / 0.3)^{2}\right] d e$. This distribution agrees well with the observed eccentricity distribution for $e \gtrsim 0.2$, but predicts too few planets at lower eccentricities, even when we exclude planets subject to tidal circularization. These findings suggest that a period of large-scale dynamical instability has occurred in a significant fraction of newly formed planetary systems, lasting 1-2 orders of magnitude longer than the $\sim 1 \mathrm{Myr}$ interval in which gas-giant planets are assembled. This mechanism predicts no (or weak) correlations between semimajor axis, eccentricity, inclination, and mass in dynamically relaxed planetary systems. An additional observational consequence of dynamical relaxation is a significant population of planets $(\gtrsim 10 \%)$ that are highly inclined $\left(\gtrsim 25^{\circ}\right)$ with respect to the initial symmetry plane of the protoplanetary disk; this population may be detectable in transiting planets through the Rossiter-McLaughlin effect.

Subject headings: planetary systems - planetary systems: formation - planets and satellites: general
\end{abstract}

\section{INTRODUCTION}

Our understanding of the origins of planetary systems has been revolutionized in the last decade by the discovery of over 250 extrasolar planets. These discoveries have vastly broadened our appreciation of the diversity of possible planetary systems, and raise a number of challenges for theories of planet formation.

One of the most important of these is the problem of large eccentricities. The median eccentricity of the known extrasolar planets is $\simeq 0.2$ (even before discarding those planets at small semimajor axes whose orbits have likely been circularized by tidal forces), a factor of two larger than that of any solar-system planet except Mercury. The largest known eccentricity is 0.93 , for HD 80606b (Naef et al. 2001). The large eccentricities are a major concern because the nearly circular, coplanar orbits of solar-system planets have been one of the strong arguments that the planets formed from a disk since the time of Kant and Laplace. The mechanism by which the extrasolar planets acquired their eccentricities, and why the eccentricities of the planets in the solar system are so much smaller than those of the known extrasolar planets (the "eccentricity problem") are still unknown. The resolution of the eccentricity problem, and the wider question of understanding the distributions and correlations of the dynamical and physical parameters of planets, are key milestones on the road towards a comprehensive theory of planet formation.

The process of planetary system formation is notoriously difficult to study theoretically. An oversimplified but useful approach is to divide it into two stages, based on the importance of the effects of gas and the protoplan-

\footnotetext{
${ }^{1}$ Department of Astrophysical Sciences, Princeton University, Princeton, NJ 08544

${ }^{2}$ School of Natural Sciences, Institute for Advanced Study, Princeton, NJ 08540
}

etary disk on the growing planets. Stage 1 lasts a few Myr, until the dissipation of the gaseous protoplanetary disk. Irrespective of the exact formation mechanism, at the end of this stage the gas giant planets should have accreted their massive envelopes - they must do so before the gas disk disappears - and some (very uncertain) number of smaller solid planetesimals, planetary embryos, and planets should also be present.

Stage 2 lasts from the end of Stage 1 until the present. In Stage 2 the evolution is driven primarily by gravitational interactions and collisions between the planets. These processes can lead to the ejection of planets into interstellar space or the Oort cloud, the consumption of planets by the host star, and collisions and mergers of planets. All of these reduce the number $N_{p l}$ of surviving planets; presumably as $N_{p l}$ declines, the system gradually evolves to a more and more stable state, and the timescale for future evolution is always comparable to the present age. While there is a vast literature on the origins of solar systems that focuses on Stage 1, investigations of Stage 2 evolution are surprisingly scarce. Yet Stage 2 is actually easier to explore than Stage 1 in most respects, since the processes in Stage 2 involve only the simplest possible physics: Newton's law of gravity and Newton's laws of motion.

The first numerical explorations of Stage 2 were motivated by the suggestion of Rasio \& Ford (1996), Weidenschilling \& Marzari (1996), and Lin \& Ida (1997) that gravitational interactions between planets could be responsible for the large eccentricities of the extrasolar planets $^{3}$. These numerical experiments demonstrated

${ }^{3}$ An additional motivation was the suggestion that hot Jupiters (massive planets on circular orbits with semimajor axes $\lesssim 0.03 \mathrm{AU}$ ) might be formed by tidal circularization of highly eccentric orbits with initial semimajor axes of a few AU (e.g. Ford et al. 2001); but, as the sample of extrasolar planets grew, this mechanism as 
that planet-planet scattering could excite eccentricities to the required levels. However, the simulations were somewhat unrealistic in that they only lasted $\sim 10^{4}$ $10^{6} \mathrm{yr}$, shorter than the likely duration of Stage 1 , and typically included only a few planets (usually two or three). Followup numerical investigations of the evolution of unstable systems containing two identical giant planets (e.g., Ford et al. 2001) found them incapable of quantitatively matching the observed eccentricity distribution, instead producing eccentricities smaller than those typically observed (see also the analytic arguments in Goldreich \& Sari 2003). However, simulations of unstable planetary systems containing two planets with unequal masses (Ford et al.|2003), or three or more planets (Marzari \& Weidenschilling 2002; Ford et al. 2003), do appear to be capable of exciting a sufficient fraction of planets to high eccentricities.

Other works have examined systems with more planets and over longer timescales. Levison et al. (1998) conducted a set of 16 long-term (1 to 16 Gyr) simulations of systems with $\sim 10$ planets, showing that stable highly eccentric orbits are a possible outcome of long-term dynamical evolution. Papaloizou \& Terquem (2001) reached a similar conclusion, although they only followed the evolution of their systems for $<10^{4}$ orbital periods, which is too short to distinguish Stage 1 from Stage 2 evolution. They also argued, following Rasio \& Ford (1996), that dynamical evolution almost inevitably leads to ejections, which could contribute to a population of free-floating planets that may be detectable by large-scale microlensing surveys. In the largest study to date, Adams \& Laughlin (2003) noted the importance of studying large ensembles of systems with similar initial conditions, and observing the evolution of ensemble averages and distributions. They traced the evolution over $10^{6} \mathrm{yr}$ of four ensembles of 100 systems each, and the evolution over $10^{7} \mathrm{yr}$ of two ensembles of 50 systems, again concluding that dynamical mechanisms are capable of producing high eccentricities, as seen in the observations.

These studies suggest that Stage 2 evolution could play an important role in determining the numbers, masses, and orbital elements of extrasolar planets, but the short durations and small ensemble sizes of these integrations limit the impact of their conclusions. They also leave many questions unanswered; in particular, the relative importance of Stage 1 and Stage 2 in determining the properties of planetary systems is still largely unexplored. An extreme (and unlikely) possibility is that the dynamical properties of planets are determined almost entirely in Stage 2, so that planetary systems are in some kind of quasi-equilibrium state that is largely independent of the initial conditions set at the end of Stage 1 - just as the Maxwell-Boltzmann distribution of gas molecules in a room is determined entirely by the temperature and total gas mass, or the distribution of

originally proposed proved unable to explain the frequency of hot Jupiters compared to planets at larger distances. However, recent work by Nagasawa et al. (2008), as well as the frequency of planetstar collisions observed in our simulations (Section 2.3), indicate that planet scattering in systems with multiple planets, or in combination with the Kozai mechanism, might still plausibly explain the observed frequencies of hot Jupiters. This possibility deserves further investigation. dark matter in a galactic halo appears to be almost independent of how the halo formed (Navarro et al. 1997). An equally extreme, and almost as unlikely, possibility is that the properties of planetary systems are determined entirely in Stage 1, so that the distribution of planetary systems looks the same at an age of a few Myr as it does after a few Gyr.

This paper is the beginning of a systematic investigation of the role of Stage 2 evolution in determining the properties of planetary systems, using accurate long-term $\left(10^{8}\right.$ yr or more) integrations of large (up to $\left.N_{\text {sys }}=1000\right)$ ensembles of mock planetary systems with up to 50 planets per system. In terms of the natural metric for the computational effort required

$$
\begin{array}{r}
P P=(\text { number of planets per system }) \times \\
\text { (number of orbital periods) } \times \\
\text { (number of systems) }
\end{array}
$$

the results presented here have $P P=5 \times 10^{12}$, a factor of 50 more than any previous exploration of Stage 2 evolution.

In this paper we focus on the dynamical evolution of the eccentricity distribution. In $\$ 2$ we briefly describe the integrator, the selection of initial conditions, and simulation results. In $\$ 3$ we discuss the distributions of eccentricities of simulated systems, their classification as "active", "inactive" and "partially active", and a comparison to observations. We quantify and justify the classification criteria in $\$ 5 . \$ 4$ discusses the inclinations of active systems, while $\sqrt{6}$ summarizes the results and discusses their implications.

\section{SIMULATIONS \\ 2.1. The Integrator}

The principal challenge in following Stage 2 evolution is the computational intensity of the problem. Reliable studies require (i) accurate numerical integrations of $\mathrm{N}$ body systems for at least $\sim 10^{8} \mathrm{yr}$ (our longer integrations show that relatively little evolution occurs between $10^{8}$ and $10^{9} \mathrm{yr}$ ); (ii) the ability to follow accurately higheccentricity orbits and close encounters between planets (a challenge that is not present in long solar-system integrations, where the planets are on well-separated, loweccentricity orbits); (iii) large ensembles of planetary systems, in order to make statistically significant predictions and explore the wide variety of possible outcomes.

We overcome these challenges by the combination of an integration strategy tailored for the problem at hand, careful hand-optimization of key subroutines of the code, and the use of large computer clusters. To efficiently simulate thousands of planetary systems in a reasonable amount of time we have decided on a "mix-and-match" strategy, infrequently switching between the BulirschStoer and hybrid symplectic integration schemes described below, depending on the conditions present in the system being integrated. Our code makes use of the publicly available integration routines of MERCURY6 ${ }^{4}$ (Chambers 1999), and extends them to support a highlevel integration scheme as follows.

Since the initial conditions of many of the simulated planetary systems produce dynamically active (numer-

\footnotetext{
4 Available at http://www.arm.ac.uk/ jec/
} 
ous close encounters, frequent scatterings to high eccentricity orbits, etc.) and short-lived systems, the integration of the first $10^{6} \mathrm{yr}$ is done using a high accuracy $\left(\epsilon=10^{-12}\right)$ conservative Bulirsch-Stoer integrator ("BS2", Chambers 1999; Press et al. 1992).

For the subsequent much longer integration $\left(10^{6}\right.$ $10^{8} \mathrm{yr}$ ), we switch to a hybrid-symplectic ("HYBRID") integrator (Chambers 1999). This integrator accurately handles close encounters between planets, without the loss of symplectic properties. It accomplishes this by introducing a Hamiltonian splitting which, in the absence of close encounters, is equivalent to the classical mixedvariable symplectic scheme (Wisdom \& Holman 1991). However, during a close encounter (defined as two planets approaching to closer than a preset number of Hill radii, usually 3 ), the HYBRID algorithm, by means of a changeover function sensitive to mutual planet separations, redistributes the (now large) perturbation due to the encountering planets to the Keplerian part of the Hamiltonian. This keeps the remaining perturbation terms small, but makes the Keplerian part analytically insoluble. The Keplerian part is therefore solved numerically to machine precision, using a high precision Bulirsch-Stoer integrator. The complete solution is advanced in this manner to the end of the close encounter, when the changeover function causes the HYBRID scheme to again become equivalent to the computationally efficient MVS. For details we refer the reader to Chambers (1999).

The HYBRID scheme handles close encounters with planets accurately, but can be susceptible to errors due to inadequate pericenter sampling in highly eccentric orbits (Rauch \& Holman 1999). To guard against this, during the symplectic integration phase the conservation of total energy and the individual orbital elements of planets in the system are continuously monitored. If the relative energy error averaged over the past 1000 timesteps, of size $h_{\text {sympl }}$, exceeds $10^{-4}$, or if the duration of pericenter passage $\left(T_{p p}\right.$, defined to be the time in which the true anomaly goes from $-\pi / 2$ to $+\pi / 2$ ) of any planet become less than 5 timesteps, the code backtracks $\Delta t=10000$ timesteps and restarts the integration using the high-accuracy BS2 integrator (an "algorithm switch"). The subsequent BS2 integration phase lasts for $\Delta t=30000 h_{\text {sympl }}$, after which we return to the hybridsymplectic integration with $h_{\text {sympl }}$ adjusted to $1 / 15$ th of the smallest value of $T_{p p}$ in the system. We call this the "HYBRID/E" scheme since it is able to handle close pericenter passages and scatterings to high-eccentricity orbits, as well as close planetary encounters.

The frequency of algorithm switches depends on the dynamical configuration of the system being integrated. As symplecticity is violated at each algorithm switch, it is desirable to keep these at a minimum. For the ensembles described in the next section, $60 \%$ of systems had no algorithm switches, $80 \%$ had fewer than 10, while only $5 \%$ had more than 100 in $10^{8}$ yr of integration.

We have tested the accuracy of this scheme by comparing the results (energies, angular momenta, and time evolution of eccentricity) of three-body integrations of highly eccentric orbits using the BS2, HYBRID, and HYBRID/E schemes (with more steps/orbit in the first two); typically, the errors of the HYBRID/E scheme are com- parable to those of HYBRID with $\sim 2$ times shorter timestep. We have also tested our algorithm by comparing the results of $10^{7} \mathrm{yr}$ integrations of systems in the n10s10 ensemble (see Table 1 and the following section) with an integration done using the high-precision Bulirsch-Stoer scheme. The final distributions of eccentricities, inclinations, semimajor axes and masses were identical, within statistical error.

\subsection{Initial Conditions}

The selection of initial conditions would ideally be based on the predictions of Stage 1 planetary formation theory; unfortunately, the theory is still too crude to allow this. We therefore picked the distributions of initial conditions (especially the eccentricities, see Figure 3) for each ensemble in a largely arbitrary fashion, with only a minimal constraint that the planets begin in some sort of a disk.

The ensemble definitions are detailed in Table 1 . We chose semimajor axes uniformly in $\log (a)$, between $a=$ 0.1 and 100 AU. Similarly, we drew the masses from a distribution uniform in $\log (M)$, between $M=0.1$ and 10 Jupiter masses (this is comparable to the observed distribution; e.g., see Tabachnik \& Tremaine 2002 and Marcy et al. 2005). For all but one ensemble we drew the eccentricity $e$ and the inclinations $I$ from a Schwarzschild distribution $^{5}$ (Binney \& Tremaine 2008):

$$
d N=S\left(x ; \sigma_{x}\right) d x=\frac{x}{\sigma_{x}^{2}} \exp \left(-\frac{x^{2}}{2 \sigma_{x}^{2}}\right) d x
$$

where $x$ is either $e$ or $I$, with $\sigma_{e}$ and $\sigma_{I}$ as given in Table 1. If an eccentricity greater than 1 is drawn, the drawing is repeated until $e<1$ is obtained. This effective truncation of the Schwarzschild distribution at $e=1$ is of practical relevance in only one ensemble (c10s40, having $\left.\sigma_{e}=0.4\right)$, as $S(x)$ falls off exponentially fast after the peak. Finally, the initial number of planets was either 3, 10 or 50 , depending on the ensemble.

The planets were approximated by homogeneous spheres of density $\rho=1 \mathrm{~g} \mathrm{~cm}^{-3}$. The central star was taken to have solar mass and radius. Planet-star collisions were allowed in all ensembles. For all but one ensemble (n10s10) planet-planet collisions were allowed as well. Both planet-star and planet-planet collisions were assumed to be fully inelastic, resulting in momentumconserving mergers with no fragmentation. We neglected all other effects, such as tidal dissipation and relativistic corrections.

We have made no attempt to constrain our initial conditions to remove systems that are unstable over short timescales, e.g., by requiring that the planets be separated by some minimum number of Hill radii. The reason is that such systems are short-lived and hence consume a negligible fraction of our computing resources.

For each ensemble, we constructed $N_{\text {sys }}$ realizations (planetary systems) which were then integrated for $10^{8} \mathrm{yr}$. This timespan corresponds to $3.2 \cdot 10^{9}$ and $10^{5}$ orbits at $a=0.1$ and $100 \mathrm{AU}$, respectively.

\subsection{The average number of planets}

\footnotetext{
${ }^{5}$ Also known as the Rayleigh distribution.
} 
TABLE 1

INITIAL CONDITIONS

\begin{tabular}{lllllccrr}
\hline Ensemble & $f(e)$ & $f(I)$ & $f(a)$ & $f(M)$ & $N_{p l}$ & $N_{\text {sys }}$ & Collisions & Class \\
\hline \hline c03s00 & 0 & $\mathrm{~S}(I ; 0.05)$ & $\mathrm{U}(\log a ;-1,2)$ & $\mathrm{U}(\log M ;-1,1)$ & 3 & 500 & yes & inactive \\
n10s10 & $\mathrm{S}(e ; 0.1)$ & $\mathrm{S}(I ; 5.73)$ & $\mathrm{U}(\log a ;-1,2)$ & $\mathrm{U}(\log M ;-1,1)$ & 10 & 1000 & no & p. active \\
c10s10 & $\mathrm{S}(e ; 0.1)$ & $\mathrm{S}(I ; 5.73)$ & $\mathrm{U}(\log a ;-1,2)$ & $\mathrm{U}(\log M ;-1,1)$ & 10 & 200 & yes & p. active \\
c10s00 & 0 & $\mathrm{~S}(I ; 0.05)$ & $\mathrm{U}(\log a ;-1,2)$ & $\mathrm{U}(\log M ;-1,1)$ & 10 & 150 & yes & p. active \\
c10s30 & $\mathrm{S}(e ; 0.3)$ & $\mathrm{S}(I ; 0.3)$ & $\mathrm{U}(\log a ;-1,2)$ & $\mathrm{U}(\log M ;-1,1)$ & 10 & 200 & yes & active \\
c50s05 & $\mathrm{S}(e ; 0.05)$ & $\mathrm{S}(I ; 0.05)$ & $\mathrm{U}(\log a ;-1,2)$ & $\mathrm{U}(\log M ;-1,1)$ & 50 & 200 & yes & active \\
c10s40 & $\mathrm{S}(e ; 0.4)$ & $\mathrm{S}(I ; 0.2)$ & $\mathrm{U}(\log a ;-1,2)$ & $\mathrm{U}(\log M ;-1,1)$ & 10 & 500 & yes & active \\
c10u80 & $\mathrm{U}(e ; 0,0.8)$ & $\mathrm{S}(I ; 3)$ & $\mathrm{U}(\log a ;-1,2)$ & $\mathrm{U}(\log M ;-1,1)$ & 10 & 500 & yes & active \\
\hline
\end{tabular}

Note. - Definition of initial conditions for the ensembles of integrations. The columns (left to right) list the name of the ensemble, the distribution functions used for drawing the initial eccentricity $f(e)$, inclination $f(I)$ (degrees), semimajor axis $f(a)$ (in AU), mass $f(M)$ (in units of Jupiter mass), the initial number of planets $N_{p l}$, and the number of realizations of the ensemble $N_{s y s}$. The column labeled Collisions specifies whether planet-planet collisions were allowed to occur during the simulation. The final column lists the ensemble classification according to the criteria of Section 3 All systems were integrated for $10^{8}$ yr. $\mathrm{S}(x ; \sigma)$ is the Schwarzschild distribution (eq. 2), $\mathrm{U}\left(x ; x_{\min }, x_{\max }\right)$ is the uniform distribution with $x_{\min } \leq x<x_{\max }$.

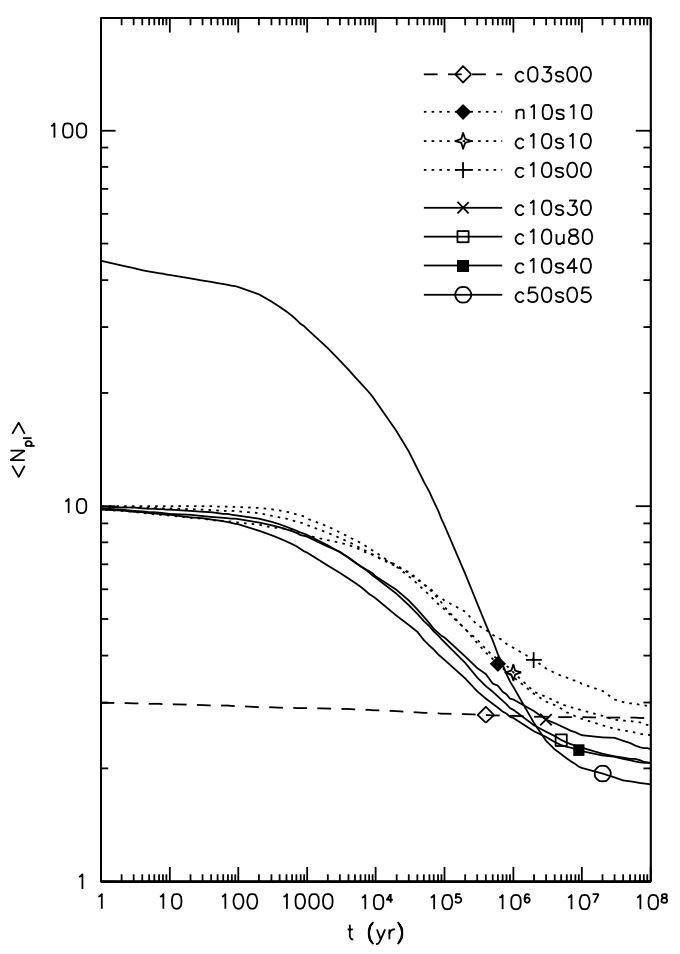

FIG. 1.- Average number of planets vs. time. Each curve shows the time evolution of $\left\langle N_{p l}\right\rangle$ for systems of a particular ensemble, as marked by different symbols.

The time history of the average number of planets per system is plotted in Figure 1, The number of planets in all but one ensemble (c03s00, which contains only three planets) exhibits a rapid dropoff, starting on a dynamical $\left(\sim-10^{3} \mathrm{yr}\right)$ and continuing on a secular timescale $\left(10^{3}\right.$ $\left.10^{5} \mathrm{yr}\right)$. A detailed breakdown, by mode of removal of planets from the system, is given in Table 2, In the first phase, the dominant mode of removal (the dominant "decay channel") is through planet-planet mergers as the randomly placed nearby planets, especially in the inner $(a<1 \mathrm{AU})$ regions, collide and merge. In the first $10^{3}$ yr between $3 \%$ and $20 \%$ of all planets are lost to planet-planet collisions. After $10^{3} \mathrm{yr}$ there are only a few collisions, with none occurring in any of the ensem-
TABLE 2

Number of Planets LOST TO EJECTIONS, MERGERS, AND STELLAR COLLISIONS

\begin{tabular}{lrrrrrrrrr}
\hline Ensemble & \multicolumn{3}{c}{$t=10^{3} \mathrm{yr}$} & \multicolumn{3}{c}{$t=10^{6} \mathrm{yr}$} & \multicolumn{3}{c}{$t=10^{8} \mathrm{yr}$} \\
& $\mathrm{E}$ & $\mathrm{M}$ & $\mathrm{S}$ & $\mathrm{E}$ & $\mathrm{M}$ & $\mathrm{S}$ & $\mathrm{E}$ & $\mathrm{M}$ & $\mathrm{S}$ \\
\hline \hline c03s00 & 0.0 & 0.1 & 0.0 & 0.1 & 0.1 & 0.0 & 0.1 & 0.1 & 0.0 \\
n10s10 & 0.5 & 0.0 & 0.3 & 5.4 & 0.0 & 1.1 & 6.2 & 0.0 & 1.3 \\
c10s10 & 0.4 & 0.5 & 0.3 & 4.0 & 0.8 & 1.6 & 4.8 & 0.8 & 1.8 \\
c10s00 & 0.3 & 1.2 & 0.2 & 3.0 & 1.5 & 1.3 & 3.9 & 1.5 & 1.7 \\
c10s30 & 0.5 & 0.7 & 0.5 & 4.2 & 0.9 & 1.9 & 4.8 & 0.9 & 2.0 \\
c10u80 & 0.5 & 0.5 & 0.7 & 4.2 & 0.6 & 2.3 & 4.8 & 0.6 & 2.5 \\
c10s40 & 1.0 & 0.7 & 0.8 & 4.3 & 0.8 & 2.1 & 4.8 & 0.8 & 2.3 \\
c50s05 & 5.7 & 9.6 & 5.1 & 27.6 & 9.7 & 9.4 & 28.9 & 9.7 & 9.5 \\
\hline
\end{tabular}

Note. - The average number of planets which by time $t$ are lost to ejections (column E), mergers through planet-planet collisions (column M) and collisions with the star (column S). In ensemble n10s10 the values in column $\mathrm{M}$ are always 0 , because planet-planet collisions are disallowed.

bles after $10^{6} \mathrm{yr}$. On $t>10^{6} \mathrm{yr}$ timescales, ejections to interstellar space become the dominant decay channel, with between $50 \%$ and $60 \%$ of planets being lost in this way $^{6}$. A further $\sim 20 \%$ are lost to collisions with the star, usually as a result of gradual eccentricity excitation by a more massive planet.

It is important to point out that our treatment of planets on such collision orbits is not complete, as we neglect the dissipative tidal forces from the host that become significant when the pericenter is $\lesssim 20$ stellar radii. In particular, dissipative tides may circularize the planet orbit at small semimajor axis before it collides with the star. This mechanism may be responsible for some, but probably not most, of the hot Jupiters. Similarly, our treatment of ejected orbits is not complete, because we neglect the tidal forces from the Galaxy that become significant when the apocenter is $\gtrsim 10^{3} \mathrm{AU}$. Galactic tides may cause some or even most planets on high-eccentricity orbits to end up in bound orbits of $\sim 10^{4} \mathrm{AU}$ (analogous to the orbits in the Sun's Oort comet cloud), rather than on unbound orbits.

In all ensembles, after approximately $10^{7} \mathrm{yr}$, the mean number of planets per system $\left\langle N_{p l}\right\rangle$ reaches an average value between 1.8 and 3 , similar to the findings of Adams \& Laughlin (2003) and Papaloizou \& Terquem (2001), despite the substantially different initial condi-

\footnotetext{
$5 \%$.

${ }^{6}$ c03s00 ensemble is an exception, with an ejection fraction of
} 


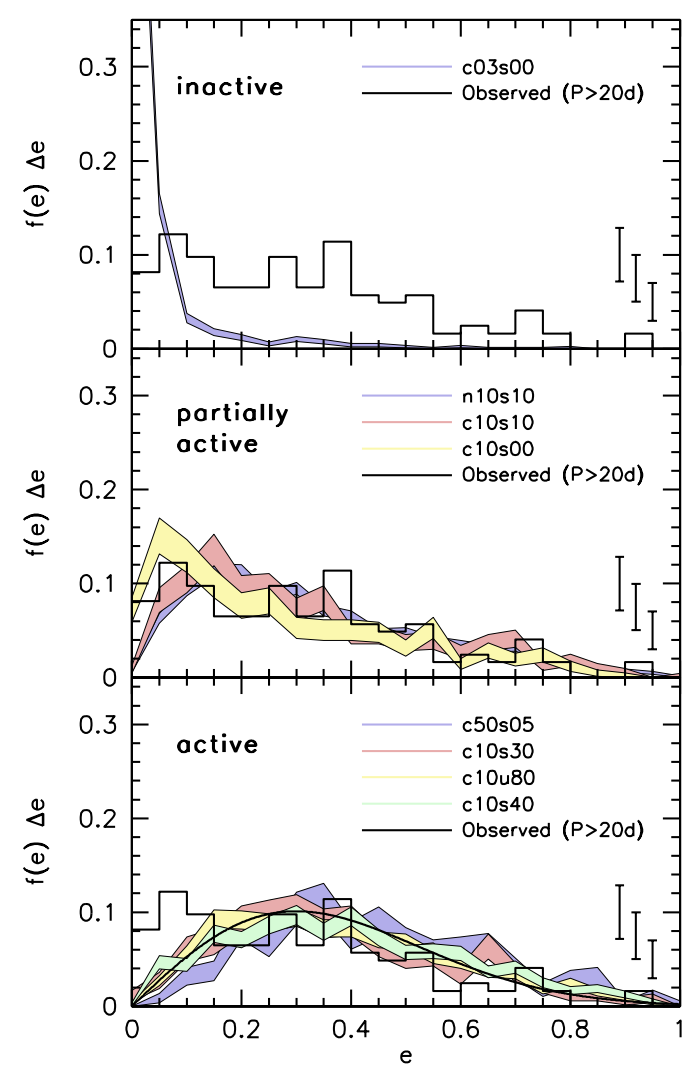

FIG. 2.- The final eccentricity distributions of the simulated ensembles. The colored bands show the final eccentricity distributions of one ensemble classified as "inactive" (top), three classified as "partially active" (middle) and four "active" ensembles (bottom). The widths of the bands illustrate the $1 \sigma$ Poisson uncertainty in the distribution due to the finite sizes of simulated ensembles. The histograms show the observed distribution of eccentricities of extrasolar planets with $P>20 \mathrm{~d}$ from Butler et al. (2006), with its typical error bars shown on the left. The bin size is $\Delta e=0.05$. Note the similarity of the final distributions of partially active and active ensembles to the observed eccentricity distribution; the excess of observed planets for $e<0.2$ in the bottom panel is discussed in 3.2 Overplotted as a smooth solid line in the bottom panel is a Schwarzschild distribution with $\sigma_{e}=0.3$.

tions (e.g., the spherical shell of Papaloizou \& Terquem 2001). Although $\left\langle N_{p l}\right\rangle(t)$ begins to level off at his point, indicating an end of strong dynamical evolution it does not do so entirely. A modest power law extrapolation near $t=10^{8} \mathrm{yr}$ still predicts a continuing decay rate of $\sim 10 \%$ of planets per decade of time.

Despite this slow continuing decay, after about $2 \times$ $10^{7}$ yr there ceases to be any significant change in the distributions of the orbital elements of the surviving planets (eccentricity, inclination and semimajor axis). After $10^{8} \mathrm{yr}$, for the purposes of this paper, the simulated ensembles can be considered to have reached an equilibrium configuration.

\section{THE ECCENTRICITY DISTRIBUTION}

\subsection{Classification of outcomes}

The panels of Figure 2 show the final $\left(t=10^{8} \mathrm{yr}\right)$ eccentricity distributions of the simulated ensembles (Table 11). To visualize the expected variance due to the fi-

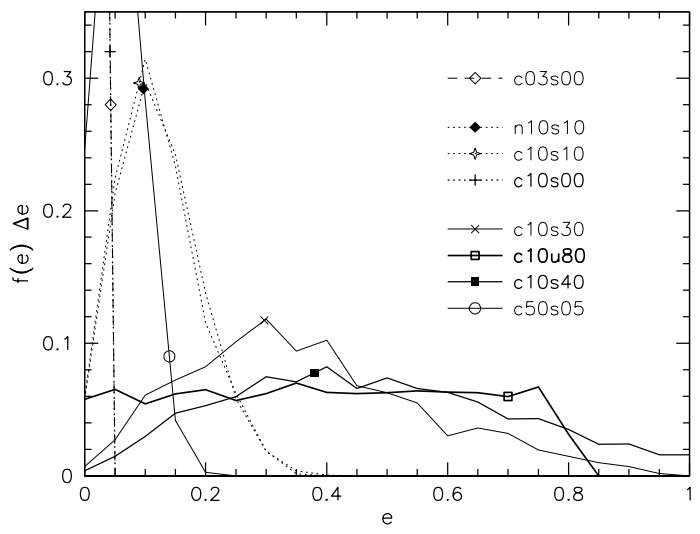

Fig. 3.- Initial eccentricity distributions of simulated ensembles. The distributions were obtained by binning the eccentricities of planets in the ensembles of Table 1 in bins of $\Delta e=0.05$. Each vertex on the plot denotes the fraction of all planets in the eccentricity bin centered on that vertex. For clarity, the vertices are connected by straight lines. Dashed, dotted and solid lines correspond to the initial eccentricity distributions of ensembles whose final distributions are shown in the top, middle and bottom panels of Figure 2 respectively.

nite number of systems in ensemble realizations, they are plotted as $\pm 1 \sigma$ wide colored bands (where $\sigma_{i}=\sqrt{N_{i}} / N$, $N$ is the total number of planets in the ensemble at $t=10^{8} \mathrm{yr}$, and $N_{i}$ is the number of planets in the $i^{\text {th }}$ bin). The solid histogram overplotted on each of the panels shows the distribution of eccentricities of 125 extrasolar planets having $P>20$ d from Butler et al. (2006), with the period condition imposed to exclude orbits that may have undergone tidal circularization. In Figure 3 we plot the corresponding initial eccentricity distributions. The dashed, dotted and solid lines in Figure 3 denote ensembles whose final distributions are shown in the top, middle and bottom plots of Figure 2, respectively.

The division into panels in Figure 2 reflects the classification of the ensembles as either inactive (top), partially active (middle), or active (bottom). This subjective division is based on two qualitative criteria: (i) the mutual likeness of the final eccentricity distributions (easily seen in case of the active ensembles), and (ii) the degree of evolution away from the initial conditions (characterized by the fraction of planets per system surviving to $t=10^{8} \mathrm{yr}$, or the change in shape from the initial to the final eccentricity distribution). The hope is that this classification reflects the degree of dynamical relaxation that the ensembles have experienced.

The first criterion clearly separates the four "active" ensembles from the others (Figure 2, bottom panel). The eccentricity distributions of these ensembles look very similar, going to zero at $e=0$ and $e=1$ and peaking around $e \sim 0.3$. Quantitatively, the medians $(0.35<\widetilde{e}<$ $0.4)$ as well as the widths ${ }^{7}(0.15<S I Q R<0.16)$ of the eccentricity distributions are all within a narrow range of values. The agreement is particularly striking for ensembles c10u80 and c50s05, considering their substantially different initial conditions (Figure 3) - note in particular that in c50s05 the typical eccentricities grow, while in c10u80 they shrink, yet both converge to a common distribution. The classification of these ensembles as ac-

\footnotetext{
${ }^{7}$ As measured from the semi-interquartile range, SIQR.
} 
tive is also consistent with the second criterion, as they have all undergone substantial dynamical evolution (e.g., as evidenced by the reduction in mean number of planets per system, Figure 1 and Table 2). All of this indicates that the final eccentricity distribution of these systems does not retain much memory of the initial conditions, and is primarily a result of dynamical relaxation. Its general features are well described by the Schwarzschild distribution of equation (2) with $\sigma_{e}=0.3$, which is overplotted as a solid curve in the bottom panel of Figure 2

The second criterion sets apart the c03s00 ensemble, the only one classified as "inactive". Its initial and final eccentricity distributions are very similar, strongly peaked at $e=0$, and inspection of the individual systems in the ensemble shows that most have not changed significantly from their initial state by the end of the integrations at $10^{8} \mathrm{yr}$. In the context of this paper, such ensembles are uninteresting as Stage 2 evolution has little effect on their properties.

We assign the remaining three ensembles to an intermediate "partially active" class. These ensembles have undergone substantial evolution, but their final eccentricity distributions are unlike those of the active systems. The difference is most pronounced at low eccentricities $(e<0.3)$, while the high-eccentricity $(e>0.3)$ tails are similar to those of the active ensembles. The described behavior could be understood as a consequence of partial relaxation. For example, it could be that there is a range of relaxation times in different systems in a partially active ensemble, so that some of the systems relax fully, while the rest retain some memory of their initial conditions. Another possibility is that the high-eccentricity tail of the distribution settles into equilibrium rapidly, while the equilibrium form for $e \lesssim 0.3$ is established more slowly. The partially active distributions can also be regarded approximately as a superposition of the distribution of fully relaxed systems, $\sim \mathrm{S}(e ; 0.3)$, and the distribution specified by the initial conditions. For our particular choice of the initial conditions, the former dominates the high eccentricities $(e \gtrsim 0.3)$, while the latter dominates for $e \lesssim 0.3$.

In $\$ 5$, we will return to the question of this classification and attempt to justify it further in a more quantitative manner.

\subsection{Comparison to observations}

We compare the simulations with the distribution of eccentricity of all planets in the Butler et al. (2006) catalog with period $P>20 \mathrm{~d}$. This catalog reflects strong selection effects, primarily in mass and semimajor axis. Selection effects should not strongly affect the eccentricity distribution since the eccentricities of the simulated planets correlate with neither mass nor semimajor axis (this will be shown in $\$ 3.5$ and 3.6 ), and since the selection effects in eccentricity are relatively small, at least in a region $(P \lesssim 5 \mathrm{yr}, e \lesssim 0.6)$ that contains most of the observed planets (Cumming 2004).

Partial qualitative agreement exists between the eccentricity distributions of the four active and three partially active ensembles and the observed extrasolar planet distribution, shown in the bottom panel of Figure 2. Despite this approximate agreement, there are obvious differences between the observed eccentricity distribution and the distribution of active systems: (i) an excess of ob- served low-eccentricity planets, and (ii) a (small) deficit of observed planets with high eccentricities. The two are inter-related, since the distribution functions $f(e)$ are normalized so that $\int_{0}^{1} f(e) d e=1$.

The excess of low-eccentricity observed planets for $e \lesssim 0.2$ in the bottom panel of Figure 2 arises because in this region the observed systems have $f(e) \sim$ const while the simulated systems have $f(e) \propto e$. Note that the linear dependence seen in the simulated systems is a generic consequence of dynamical relaxation: in a relaxed distribution, there is no reason to expect a singularity in phase-space density at $e=0$ and therefore the dependence $f(e) \propto e$ is simply due to the smaller volume of phase space available near the origin (the radial action $J_{r} \propto e^{2}$ at fixed semimajor axis so $\left.d J_{r} \propto e d e\right)$. Thus the excess of observed planets at low eccentricities is presumably due to systems that have not experienced the period of dynamical activity that our active-ensemble simulations describe.

On the other hand, the simulations are highly successful in reproducing the mid- and high-eccentricity part of the observed distribution $(e \gtrsim 0.2)$. They all successfully reproduce the peak of the observed distribution near $e \sim 0.3$, as well as the decline towards $e=1$ and the general shape of this decline. They appear to predict somewhat more high-eccentricity planets than are observed, even when the $e<0.2$ excess is taken into account, but the excess is only marginally statistically significant and also is consistent with the effects of observational bias. In particular, Cumming (2004) showed that while the detection efficiency of radial velocity surveys is roughly constant for planets with $e \lesssim 0.6$, it drops rapidly beyond $e \sim 0.6$. We have conducted simple tests, in which we apply a linearly or quadratically decreasing detection efficiency $^{8}$ to simulated data at $e>0.6$, and find that the correction works in the direction of improving the agreement with our simulations. But overall, its effect on the shape of the observed distribution is negligible for the current data.

We quantify the discussed similarities and differences using Figure 4 and Tables 3 and 4 . Figure 4 compares the final cumulative eccentricity distributions of the active ensembles, the observed distribution, and the Schwarzschild distribution $\mathrm{S}\left(e ; \sigma_{e}=0.3\right)$ which captures the general features of the simulated distributions. The comparison is made both over the entire range of eccentricities (top panel), and restricted to the range $e>0.2$ to exclude possible contamination by inactive systems in the observed sample (bottom panel). Table 3 shows the $p$-values of the Kolmogorov-Smirnov ${ }^{9}$ statistic calculated between pairs of all active ensembles, the observed distribution, and the $\mathrm{S}\left(e ; \sigma_{e}=0.3\right)$ distribution. Table 4 shows the same statistic for partially active ensembles.

At a $5 \%$ significance level ${ }^{10}$ the eccentricity distri-

${ }^{8} p_{\text {det }}=1-x$ and $p_{\text {det }}=1-x^{2}$, where $x=(e-0.6) / 0.4$.

9 The same tests were repeated using Pearson's $\chi^{2}$ statistic and led to qualitatively similar conclusions. However, for these data sets the $\chi^{2}$ statistic has less distinguishing power than the KS statistic (typical $p$-values were a factor of two larger).

10 In this paper we adopt the $\alpha=0.05$ significance level as the threshold for rejecting the null hypothesis that two samples were drawn from the same distribution. That is, if at least $5 \%$ of pairs of samples randomly drawn from a given distribution would differ by more than the observed amount as measured by their KS statistic, 


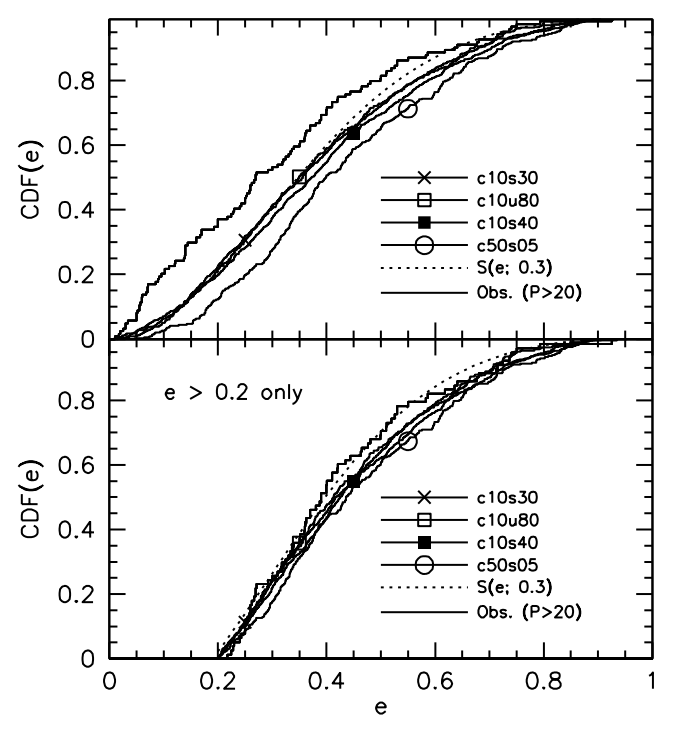

FIG. 4.- The cumulative distribution functions of eccentricity in the observed sample (solid line with no symbol) and the four active ensembles (solid lines with symbols), and the cumulative distribution function of the Schwarzschild distribution $\mathrm{S}(e ; 0.3)$ (eq.2 dotted line) for all planets (top) and planets with $e>0.2$ (bottom).

TABLE 3

KS TESTS OF ECCENTRICITY DISTRIBUTIONS (ACTIVE ENSEMBLES)

\begin{tabular}{lccccc} 
All & $\mathrm{c} 10 \mathrm{u} 80$ & $\mathrm{c} 10 \mathrm{~s} 40$ & $\mathrm{c} 50 \mathrm{~s} 05$ & Observed & $\mathrm{S}(e ; 0.3)$ \\
\hline \hline c10s30 & 0.99 & 0.42 & $9 \times 10^{-4}$ & $5 \times 10^{-3}$ & 0.41 \\
c10u80 & $\cdot$ & 0.21 & $3 \times 10^{-5}$ & $2 \times 10^{-3}$ & 0.15 \\
c10s40 & $\cdot$ & $\cdot$ & $3 \times 10^{-3}$ & $5 \times 10^{-4}$ & $8 \times 10^{-4}$ \\
c50s05 & $\cdot$ & $\cdot$ & $\cdot$ & $5 \times 10^{-7}$ & $2 \times 10^{-5}$ \\
Observed & $\cdot$ & $\cdot$ & $\cdot$ & $\cdot$ & $2 \times 10^{-4}$ \\
\hline & & & & & \\
$e>0.2$ & c10u80 & c10s40 & c50s05 & Observed & $\mathrm{S}(e ; 0.3)$ \\
\hline \hline c10s30 & 0.99 & 0.57 & 0.28 & 0.54 & 0.21 \\
c10u80 & $\cdot$ & 0.57 & 0.14 & 0.30 & 0.02 \\
c10s40 & $\cdot$ & $\cdot$ & 0.48 & 0.13 & $1 \times 10^{-4}$ \\
c50s05 & $\cdot$ & $\cdot$ & $\cdot$ & 0.07 & $2 \times 10^{-4}$ \\
Observed & $\cdot$ & $\cdot$ & $\cdot$ & $\cdot$ & 0.53 \\
\hline
\end{tabular}

Note. - The $p$-values of Kolmogorov-Smirnov tests between the eccentricity distributions of active ensembles (c10s30, c10u80, c10s40 and c50s05), the observed sample of extrasolar planets with $P>20$ d ("Observed"), and the Schwarzschild distribution with $\sigma_{e}=0.3$ (last column). When testing against the $\mathrm{S}(e ; 0.3)$ distribution the null hypothesis is that the sample was drawn from it (one-sample KS test). Everywhere else, the null hypothesis is that the pair of samples being tested was drawn from the same (but unspecified) underlying distribution (two-sample KS test). The top set of rows shows the results when planets of all eccentricities are included in the test. The bottom set shows the results for the subsample with $e>0.2$.

butions of three of the four active ensembles (c10s30, c10u80, c10s40) are pairwise ${ }^{11}$ consistent with being drawn from the same eccentricity distribution (Table 3),

we conclude that the hypothesis cannot be rejected.

11 We use the term "pairwise" to emphasize that these are twosample tests, and that they do not test whether all ensembles are simultaneously consistent with being drawn from the same underlying distribution. For example, three ensembles $\mathrm{A}, \mathrm{B}$ and $\mathrm{C}$ with two-sample KS statistic $p$-values $p(A B), p(B C)$, and $p(A C)$ above a significance level $\alpha$ may produce $p(A B C)<\alpha$ in a three-sample test.
TABLE 4

KS TESTS OF ECCENTRICITY DISTRIBUTIONS (PARTIALLY ACTIVE ENSEMBLES)

\begin{tabular}{lcccc} 
All & c10s10 & c10s00 & Observed & $\mathrm{S}(e ; 0.3)$ \\
\hline \hline n10s10 & 0.11 & 0 & 0.15 & 0 \\
c10s10 & $\cdot$ & $7 \times 10^{-6}$ & 0.32 & 0 \\
c10s00 & $\cdot$ & $\cdot$ & 0.01 & 0 \\
Observed & $\cdot$ & $\cdot$ & $\cdot$ & \\
\hline & & & & \\
$e>0.2$ & c10s10 & c10s00 & Observed & $\mathrm{S}(e ; 0.3)$ \\
\hline \hline n10s10 & 0.75 & 0.55 & 0.72 & $1 \times 10^{-3}$ \\
c10s10 & $\cdot$ & 0.55 & 0.80 & 0.06 \\
c10s00 & $\cdot$ & $\cdot$ & 0.68 & 0.01 \\
Observed & $\cdot$ & $\cdot$ & $\cdot$ & 0.53 \\
\hline
\end{tabular}

Note. - Analogous to Table 3 but for partially active ensembles.

despite their quite different initial conditions (Table 1). The smallest $p$-values are obtained in tests involving c50s05, which began with 50 planets per system, compared to 10 for the other active ensembles. This difference may point to a dependence of the finer details of the final eccentricity distribution on the initial conditions. When restricted to the subsample with $e>0.2$, the distributions of all active ensembles are pairwise consistent. Analogous tests of the partially active ensembles (Table 4) reveal similar results: substantial differences in the eccentricity distributions of the full samples but consistency among the subsamples with $e>0.2$.

Using the same statistic, we compare the final $e>0.2$ distributions of active and partially active ensembles with the observations (Tables 3 and 4 , the columns and rows labeled "Observed"). In all cases, the simulated $e>0.2$ distributions and the observed distribution are consistent with being drawn from the same underlying distribution at the $5 \%$ significance level. We interpret this as evidence that the high-eccentricity component $(e>0.2)$ of active and partially active ensembles is populated by planets from dynamically relaxed systems, as is the majority of the high-eccentricity component of the observed extrasolar planet population ${ }^{12}$. Taking into account the simplifying approximations of our simulation (giant planets only, no debris, gas disk or any other influences, no binary companions), the exclusion of all nongravitational effects (e.g., tidal effects), and the likely differences between our assumed initial distribution of masses and orbital elements and the actual distribution, the agreement obtained for $e>0.2$ is quite remarkable.

The Schwarzschild distribution $\mathrm{S}\left(e ; \sigma_{e}=0.3\right)$ provides an approximate qualitative representation of the eccentricity distribution of the active ensembles (Figure 2 bottom panel) but is not quantitatively consistent with several of them according to the KS statistic - probably we could improve the consistency by fitting $\sigma_{e}$ to the eccentricity distributions, but this would provide only an illusion of greater accuracy.

In the scheme introduced in 83.1 , the ensemble of observed planets would be classified as partially active. Its distribution of eccentricities may be decomposed into two components. One, resulting from dynamical relaxation, dominates the $e>0.2$ regime, contains $75 \%$ of $P>20 \mathrm{~d}$

12 Other mechanisms, such as Kozai oscillations due to a companion(Wu \& Murrav 2003; Fabrvcky \& Tremaine 2007), may be responsible for a minority of high-eccentricity planets. 


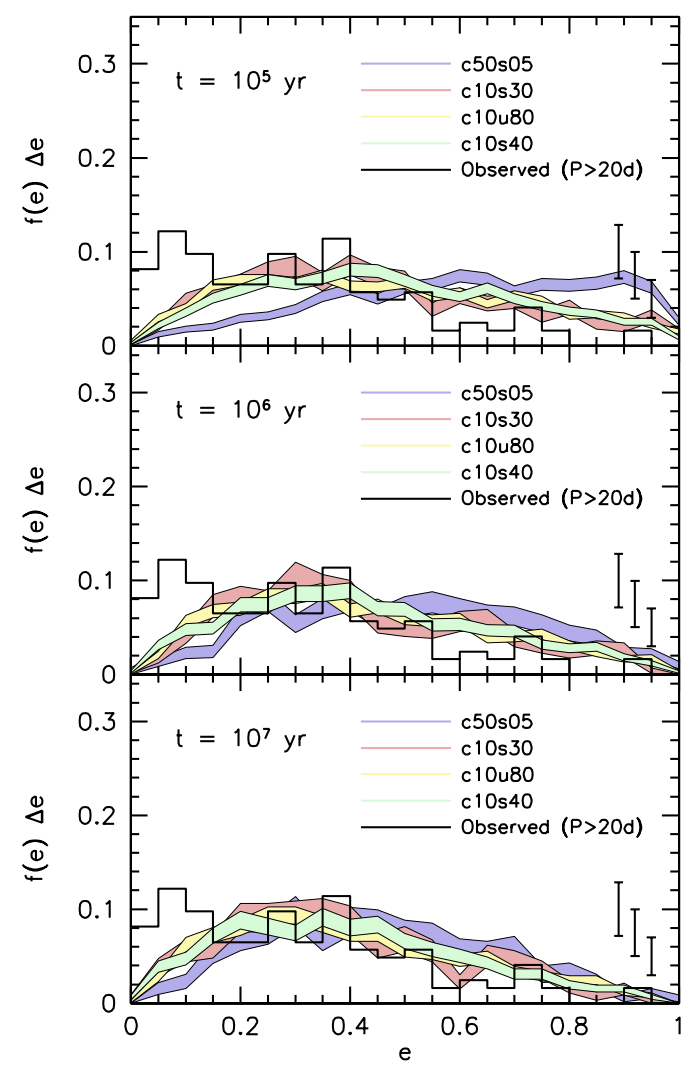

FIG. 5. - Time evolution of eccentricity distributions of active ensembles. The top, middle, and bottom panel show the eccentricity distributions at $t=10^{5}, 10^{6}$ and $10^{7} \mathrm{yr}$ respectively. The meaning of symbols is the same as in Figure 2 where the corresponding distributions at $10^{8} \mathrm{yr}$ are shown in the bottom panel.

(or $55 \%$ of all) planets, and agrees well with the $e>0.2$ distributions of the active and partially active ensembles in our simulations. The other contains $25 \%$ of $P>20 \mathrm{~d}$ (or $45 \%$ of all) planets and dominates the $e<0.2$ regime; here the eccentricities were set by other processes, possibly the (unknown) initial conditions or damping by lowmass planets, planetesimals, or residual gas. With this decomposition in mind, for the remainder of this paper we will mostly concentrate on the properties of the relaxed component (the active ensembles).

\subsection{Time evolution}

The panels of Figure 5 show the time evolution of the eccentricity distributions of active ensembles from $t=10^{5} \mathrm{yr}$ (top) to $t=10^{7} \mathrm{yr}$ (bottom). At $t=10^{5} \mathrm{yr}$ the distributions are still dissimilar, especially that of the c50s05 ensemble which is still undergoing strong dynamical activity (a consequence of the larger initial number of planets). By $t=10^{6} \mathrm{yr}$ the fraction of high eccentricity planets has been reduced in all ensembles, with a simultaneous increase in the frequency of planets of moderate eccentricity. At $t=10^{7} \mathrm{yr}$ the eccentricity distributions have largely converged to a common characteristic shape, with the biggest change from $10^{7}$ to $10^{8} \mathrm{yr}$ being a further reduction in the number of high eccentricity planets, primarily by ejections (see bottom panel of Figure 21).

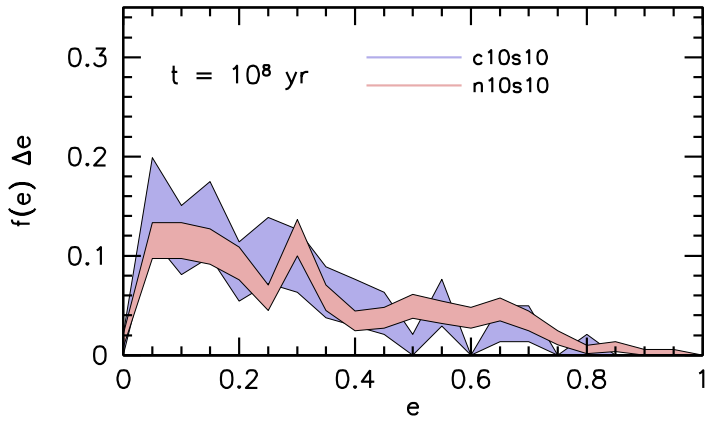

FIG. 6.- Comparison of eccentricity distributions of planets having $a<1 \mathrm{AU}$ at $t=10^{8} \mathrm{yr}$, in ensembles n10s10 and c10s10. The meaning of symbols is the same as in Figure 2

By comparing ensembles n10s10 and c10s10 we tested the influence of planet-planet collisions on the shape of the final eccentricity distribution. These two ensembles share the same initial conditions, except that in the case of c10s10 collisions were allowed to occur, while for n10s10 they were not (i.e., the planets were assumed to have density $\rho=1 \mathrm{~g} \mathrm{~cm}^{-3}$ in the first case, and to be point masses in the second). We found no significant difference in the outcomes of these two cases (Figure 2 , middle panel), and the final eccentricity distributions are consistent with being drawn from the same distribution at the $10 \%$ significance level (Table 4). Since the effects of planet-planet collisions are likely to be most noticeable at small semimajor axes, we also compared the distributions of n10s10 and c10s10 planets having semimajor axis $a<1$ AU (Figure 6), but again found no significant difference.

This outcome was not unexpected, since we have already observed that the final eccentricity distribution is established over long timescales $\left(\sim 10^{5}-10^{8}\right.$ yr, Figure [5), while planet-planet collisions are infrequent events (Table 2) which preferentially occur early $\left(t \lesssim 10^{4} \mathrm{yr}\right)$. Physically, the ratio of the escape speed from the planets to the typical encounter speeds between planets at $1 \mathrm{AU}$ is large enough that the planets act like point masses.

\subsection{Dependence on semimajor axis}

Figures $[7$ and 8 examine the semimajor axis distribution and the correlation of eccentricity with semimajor axis in the four active ensembles.

The initial semimajor axis distribution of all of our ensembles was uniform in $\log a$. The final distribution in the active ensembles shows depletion at low $a$ and some spreading beyond $100 \mathrm{AU}$ (the initial outer limit). Neither is particularly surprising given that interactions are strongest at small semimajor axes (thus the depletion at low $a$ ), and that planet-planet scattering tends to spread out the semimajor axis distribution. The efficiency of depletion is particularly striking in case of c50s05, where fewer than $2 \%$ of planets remain on orbits closer than $a=1 \mathrm{AU}$; nevertheless, in general it appears that the semimajor axis distribution in active systems retains a strong memory of the initial conditions. 


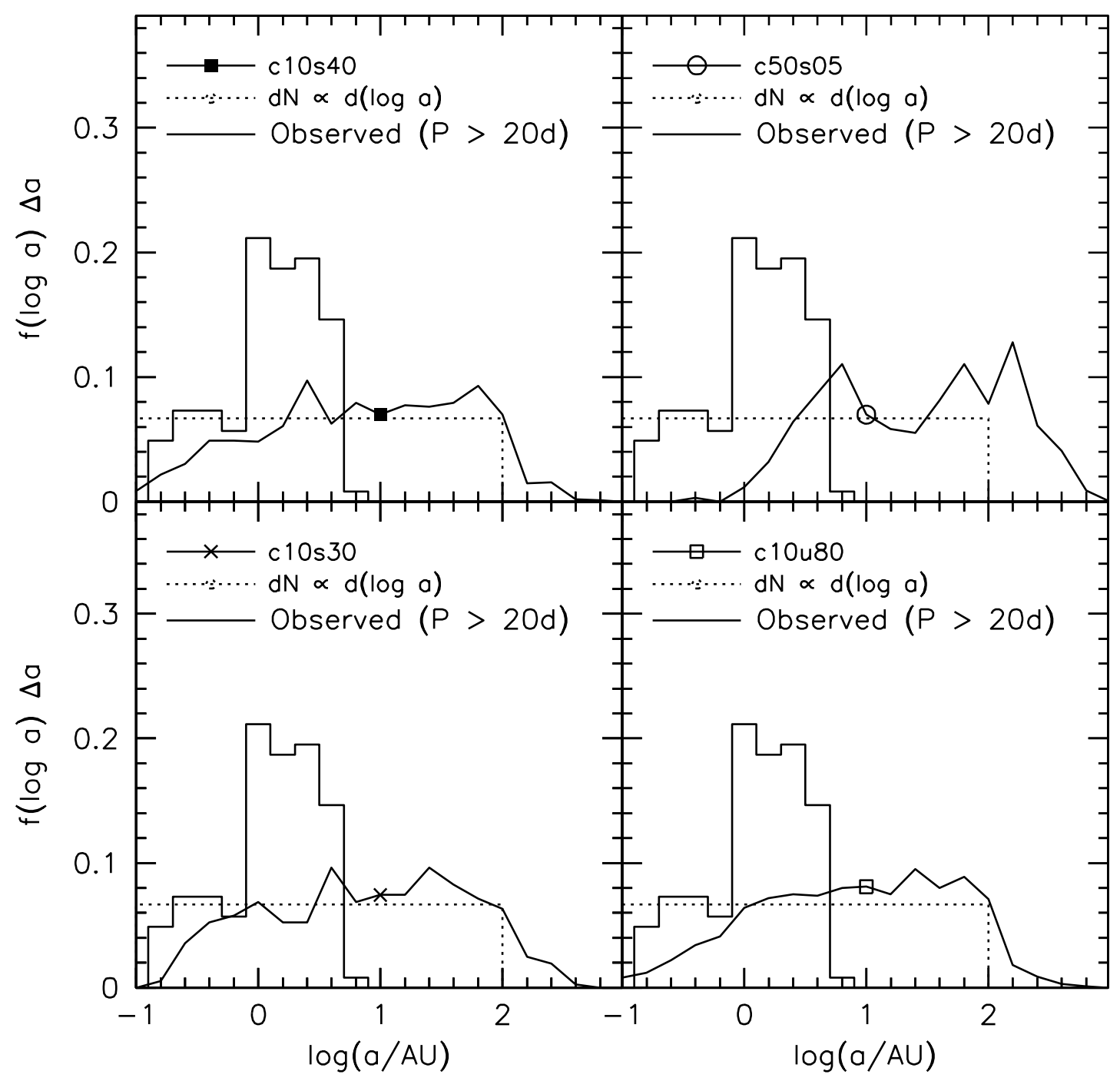

FIG. 7.- The distribution of semimajor axes in the four active ensembles. The semimajor axes of known exoplanets having $P>20 \mathrm{~d}$ are plotted as a histogram. Note that the two distributions are not directly comparable, due to the significant observational biases existing in the latter. The bin size is $\Delta \log a=0.2$.

Of greater consequence for comparisons of the eccentricity distributions with the observations is the correlation of eccentricity and semimajor axis. We find no significant $e-a$ correlation, except for a small subset (less than $5 \%$ of the total) of planets scattered to higheccentricity orbits at $a>100 \mathrm{AU}$ (Figure 8); this correlation is expected, since such orbits are likely to result from close encounters with planets at smaller radii, and hence must have pericenters $q \lesssim 100$ AU so $a=q /(1-e)$ is correlated with $e$. The widths of the eccentricity distributions at fixed $a$ remain approximately constant (semiinterquartile range $\mathrm{SIQR} \simeq 0.15$ ) over the entire range of semimajor axes, indicating that the shape of the eccentricity distribution does not appreciably vary with $a$ either. This has already been implicitly assumed in $\$ 3.2$ where we compared the distributions of the entire simulated sample (with median $\widetilde{a} \sim 7.5 \mathrm{AU}-8.5 \mathrm{AU}$ in c10ensembles and $\widetilde{a} \sim 34 \mathrm{AU}$ in c50s05) to the observed sample $(\widetilde{a} \sim 1.3 \mathrm{AU})$.

\subsection{Mass-eccentricity correlations}

In Figure 9 we show the final $\left(t=10^{8} \mathrm{yr}\right)$ distributions of $M \sin I$ in the four active ensembles (solid lines), compared to the observed $M \sin I$ distribution (solid histograms) and the initial mass distribution function $\left(d N \propto M^{-1} d M\right.$, dotted line). To obtain $M \sin I$ from simulations, we assume that the orbit normals are uniformly and randomly distributed on a sphere and assign the inclinations accordingly. Note that the observed distribution of exoplanet $M \sin I$ is heavily biased by the difficulty of detecting low-mass planets; the true distribution almost certainly is steeper and extends to lower values than the measured one.

The mass distributions for the three ensembles that began with $N_{p l}=10$ planets per system (c10s40, c10s30, and c10u80) converge to a similar final shape by $t=$ $10^{8} \mathrm{yr}$. The $M \sin I$ distribution of c50s05 (started with $N_{p l}=50$ planets per system) is different: strongly 


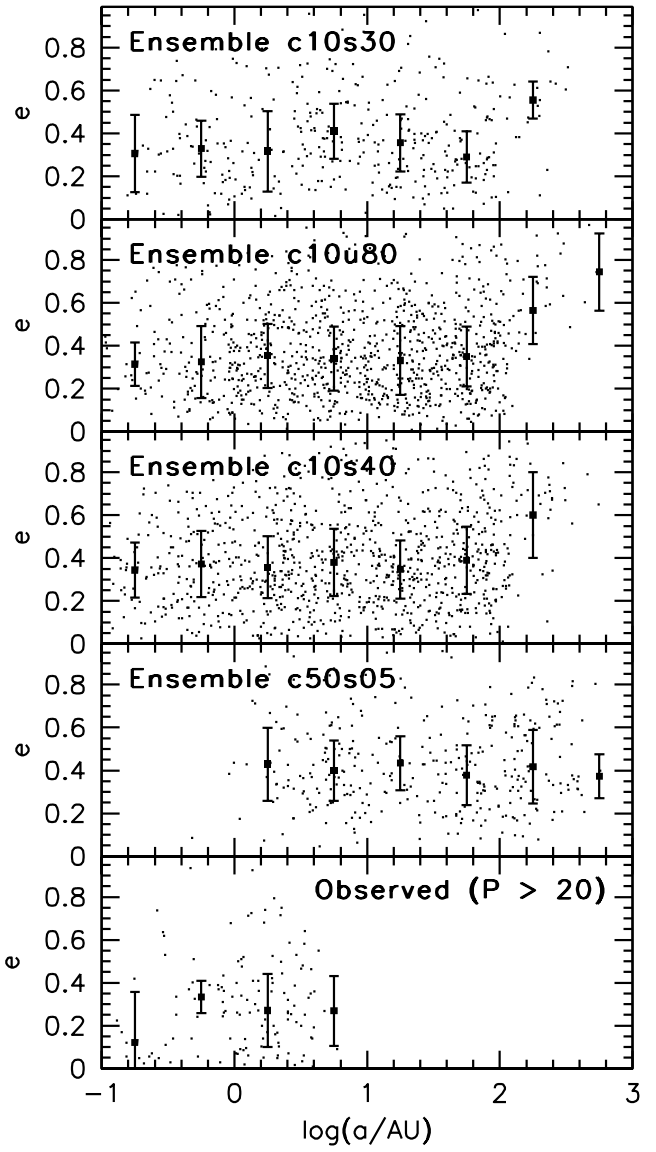

FIG. 8.- The correlation of semimajor axis and eccentricity of active ensembles (top four panels) and the observed $(P>20 \mathrm{~d})$ sample (bottom panel). The error bars show the semi-interquartile range.

peaked around $M \sin I \sim 7.5 M_{J}$, with an extended tail beyond $M \sin I=10 M_{J}$ due to a significantly higher fraction of mergers than the other ensembles (Table 2).

Relative to the initial conditions (dotted line), all of these simulations show a sharp reduction in the fraction of planets with small masses $\left(M \lesssim M_{J}\right)$, arising because low-mass planets are more readily removed from the systems, and mergers shift the distribution towards higher masses. Compared to the observed distribution, these three ensembles show a statistically significant deficit of planets in the range $M_{J} \lesssim M \sin I \lesssim 3 M_{J}$ but agree with the observed distribution for $M \sin I \gtrsim 3 M_{J}$ (KS test; $5 \%$ significance level). The difference at low masses is not due to selection effects in the observed sample, since these preferentially delete the lower mass planets, not the higher mass ones. Simulations using a steeper initial mass distribution may result in a better fit to the observational data.

Figure 10 shows the correlation of eccentricity and $M \sin I$ at $t=10^{8} \mathrm{yr}$ in the four active ensembles and in the observed sample. We find non-zero but statistically insignificant positive mass-eccentricity correlations in the simulated ensembles. The median eccentricity of planets in different mass bins is roughly constant $(\widetilde{e} \sim 0.35)$, as is the width $e_{\mathrm{SIQR}} \sim 0.15$ of the eccentricity distribu- tions as measured by the semi-interquartile range. The lack of strong correlation between $e$ and $M \sin I$ may be surprising given that one might expect some kind of equipartition, in which the most massive planets acquire the smallest eccentricities in planet-planet scattering. Indeed, as we show in Figure [1] such correlations are present at some times during the simulation, although they are never as strong as equipartition of radial energies would predict.

In the panels of Figure 11] we show the evolution of the mass-eccentricity correlation for one ensemble (the other active ensembles exhibit similar behavior). The top left panel shows the dependence of average eccentricity on mass at $t=0$, and the subsequent panels (left to right, top to bottom) show the $e$ vs. $M \sin I$ correlation at $t=10^{5}, 10^{6}, 10^{7}$, and $10^{8} \mathrm{yr}$. The correlation of mass and eccentricity changes during the integration. Initially $\left(0<t<10^{5} \mathrm{yr}\right)$, the median eccentricity of low-mass planets grows, with the median eccentricity of high-mass planets also growing but by a smaller amount. This is followed by a period of decline of the median eccentricity of both low- and high-mass planets $\left(10^{5}<t<10^{7} \mathrm{yr}\right.$ in Figure [11), with eccentricities evolving to a massindependent median value $\widetilde{e} \sim 0.35$ at $t>10^{7} \mathrm{yr}$.

This behavior is a consequence of dynamical interactions in the system. At early times $\left(0<t<10^{5} \mathrm{yr}\right)$, the low-mass planets are easily excited to higher eccentricity orbits by their massive counterparts. As these high-eccentricity planets are gradually removed from the system (through close encounters, ejections, or collisions with the star) the average eccentricity of the remaining planets decreases. This is supported by the finding that $\sim 80 \%$ of planets that are excited to $e>0.6$ are removed from the system by $10^{8} \mathrm{yr}$. The median eccentricity of the high-mass planets initially increases, and then gradually decreases over time through the removal of planets on high-eccentricity orbits and the damping of eccentricity by lower mass planets. These processes continue until enough planets are removed from the system and the orbits of the remaining planets become sufficiently separated, thus rendering the system stable for the remainder of the integration.

The observed sample (Figure 10, bottom panel) shows a positive correlation between mass and eccentricity at the $\sim 5.5 \sigma$ level. This signal comes largely from a difference in median eccentricity between the planets with $M \sin I \lesssim M_{J}$ and those with $M \sin I \gtrsim 3 M_{J}$. If the sample is restricted to the subset with $\widetilde{M} \sin I>M_{J}$, its significance drops to $\sim 2.8 \sigma$. Some of this correlation may result from selection effects (planets are harder to detect either if the mass is low or the eccentricity is high), so we prefer to wait for more data before investigating the implications of a possible mass-eccentricity correlation.

\section{INCLINATIONS OF ACTIVE SYSTEMS}

Cumulative and differential distributions of inclinations in active ensembles are shown in Figure 12. The left column shows the distribution of inclinations relative to the symmetry plane of the initial conditions $\left(I_{R}\right)$, while on the right the inclinations are computed relative to the invariable plane (the plane perpendicular to the total angular momentum) of each system at the end of the simulation $(I)$. 


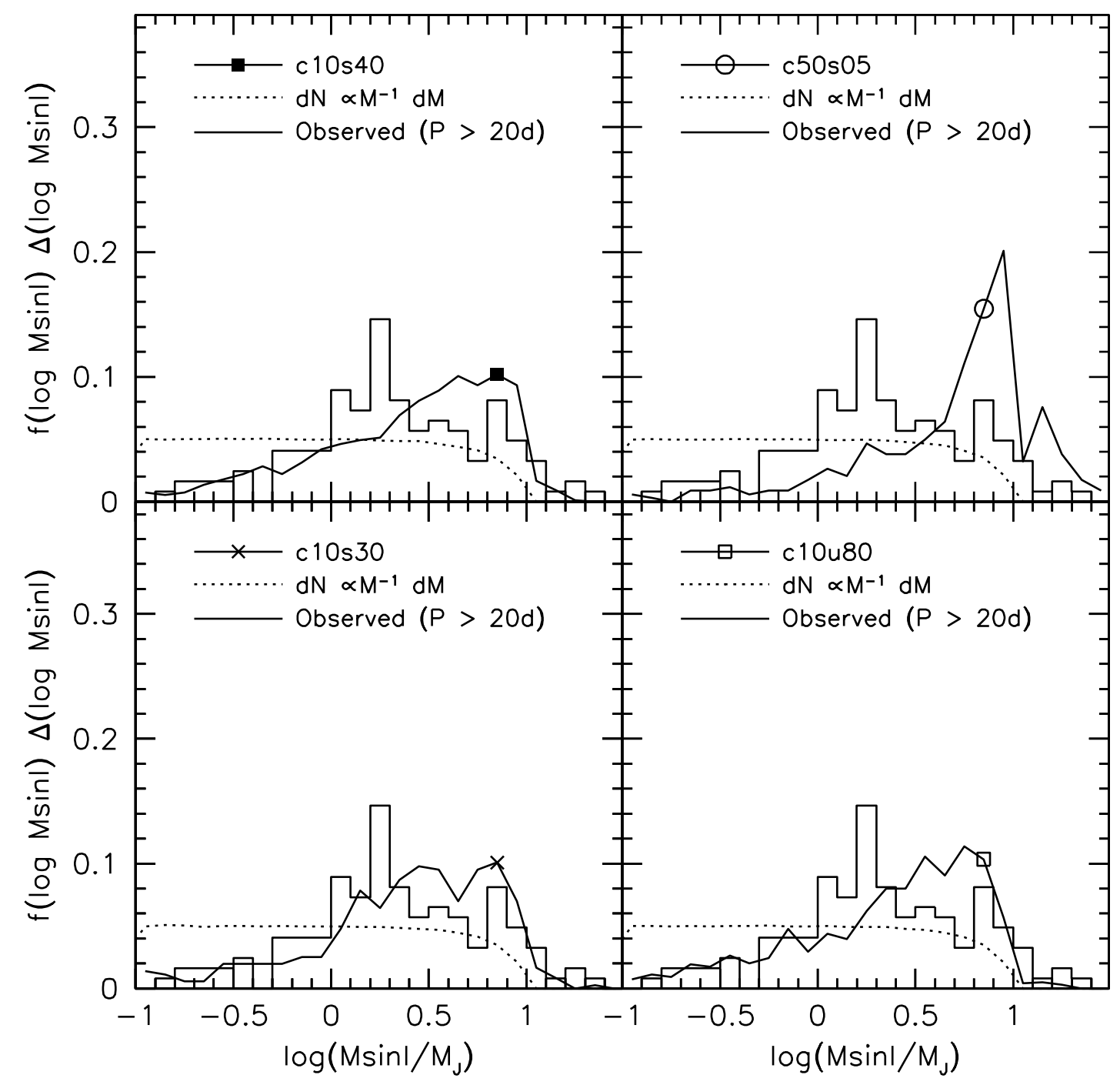

FIG. 9.- Distribution of $M \sin I$ at $t=10^{8} \mathrm{yr}$ in the four active ensembles (solid line), compared to the observed distribution of $M$ sin $I$ (solid histogram), and the distribution of initial conditions $\left(d N \propto d \log M,-1 \leq \log \left(M / M_{J}\right) \leq 1\right.$; dotted line). To obtain $M$ sin $I$ in the simulations we assume that the orbit normals are uniformly distributed on a sphere. The bin $\operatorname{size}$ is $\Delta \log (M \sin I)=0.1$.

The inclinations $I$ referred to the invariable plane are in principle measurable by precision astrometry of multiplanet systems. Such measurements are currently out of reach of ground-based telescopes, but should become feasible with the launch of the SIM PlanetQuest mis$\operatorname{sion}^{13}$. On the other hand, if the symmetry axis of the initial conditions is identified with the axis of stellar rotation, the inclinations $I_{R}$ can be identified with stellar spin-planetary orbit misalignments $\lambda$ and are measurable for transiting planets using the Rossiter-McLaughlin effect (the RM effect; e.g., Ohta et al. 2005; Winn et al. 2005). Other mechanisms, such as Kozai oscillations plus tidal friction, can also cause spin-orbit misalignment (Fabrycky \& Tremaine 2007).

The final inclination distributions of the three active ensembles that start with 10 planets appear similar, at least for $I_{R} \gtrsim 4^{\circ}$, with medians $7^{\circ}<\widetilde{I}_{R}<9^{\circ}$

13 http://planetquest.jpl.nasa.gov/SIM/sim_index.cfm
$\left(4^{\circ}<\widetilde{I}<6^{\circ}\right)$. For the 50 planet ensemble c50s05, the inclinations are larger (median $\widetilde{I}_{R}=19^{\circ}$ and $\widetilde{I}=10^{\circ}$ ) and the shape of the distribution is different. All ensembles have a significant fraction of planets at high inclinations at the end of the integrations; $10 \%$ of planets of the c10ensembles possess inclinations $I_{R} \gtrsim 25^{\circ}\left(I \gtrsim 20^{\circ}\right)$, while $10 \%$ of c50s 05 planets are inclined by $I_{R}>51^{\circ}\left(I>40^{\circ}\right)$. There is no strong correlation of inclination and eccentricity (Figure 13) except for the most eccentric planets $(e>0.7)$. A weak correlation exists with mass (Figure 14), in the sense that the inclinations of less massive planets are more easily excited than those of more massive ones. Inclinations are weakly correlated with the semimajor axis (Figure 15), in the sense that the inclinations of inner planets are on average higher than those of the outer. The strongest dependence is seen for ensemble c10s30 $\left(d \widetilde{I}_{R} / d \log a=-2.8 \mathrm{deg} / \mathrm{dex}\right)$, while the effect is weakest for $\mathrm{c} 10 \mathrm{~s} 40\left(d \widetilde{I}_{R} / d \log a=-0.9 \mathrm{deg} / \mathrm{dex}\right)$. 


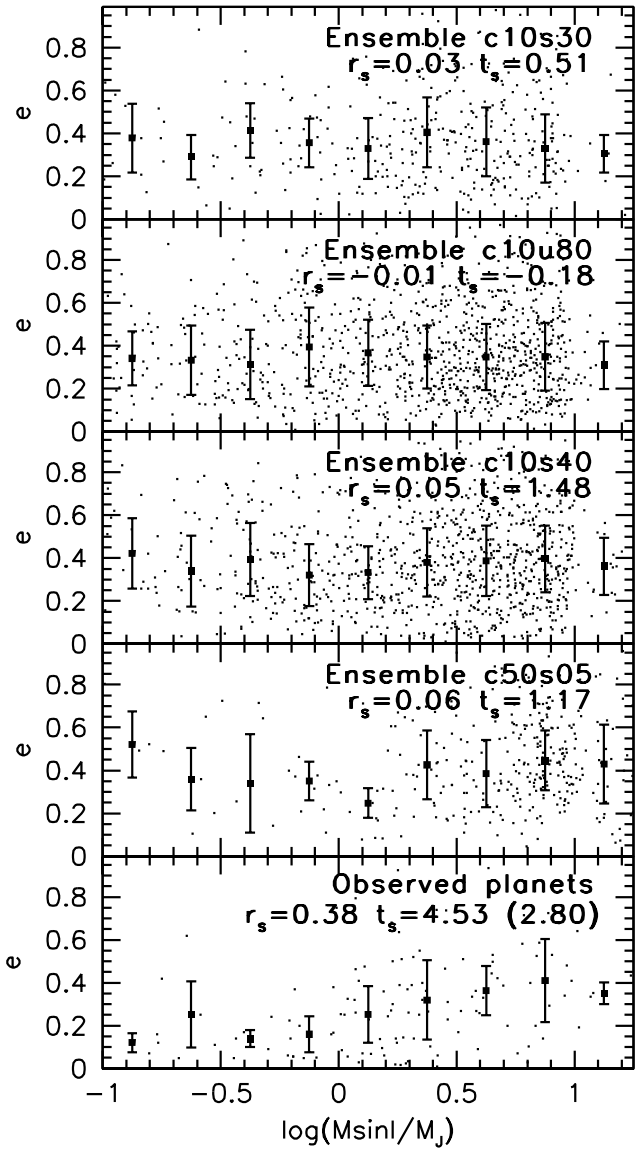

FIG. 10.- Dependence of eccentricity on $M \sin I$ at $10^{8} \mathrm{yr}$ for the four active ensembles (top four panels) and the observed $(P>20 \mathrm{~d})$ planets. Dots mark the eccentricity and $M \sin I$ of each planet. To obtain $M \sin I$ in the simulations we assume that the orbit normals are uniformly distributed on a sphere. The median and semi-interquartile ranges of $e$, calculated in bins of size $\Delta \log (M \sin I)=0.25$, are marked by the solid squares and error bars, respectively. The values of the Spearman rank correlation coefficient $r_{s}$ evaluated over all planets in the sample and the corresponding normally distributed $N(\mu=0, \sigma=1)$ variable $t_{s}$ are given in the upper right corner of each panel. The number in parentheses in the bottom panel is the value of $t_{s}$ for a subsample satisfying $M \sin I>M_{J}$.

Until recently, all measurements of the projected angle $\lambda$ between the stellar spin axis and the planetary orbit axis from the RM effect were either small $\left(\leqslant 5^{\circ}\right)$ or consistent with zero (Queloz et al. 2000; Winn et al. 2005, 2006; Narita et al. 2007a; Wolf et al. 2007). While most of these involved (possibly tidally evolved) hot Jupiters, no misalignment was found even in the case of the significantly eccentric HAT-P-2b $(e=$ 0.5; Bakos et al. 2007; Winn et al. 2007; Loeillet et al. 2007). A recent exception is HD $17156 \mathrm{~b}(P=21.2 \mathrm{~d}$, $e=0.67$, Fischer et al. 2007; Barbieri et al. 2007), for which Narita et al. (2007b) report a possible spin-orbit misalignment of $\lambda=62^{\circ} \pm 25^{\circ}$. At the nominal value $\lambda=62^{\circ}$ this planet would be unusual in the context of our simulations (a 1/100 event in the c10- ensembles and a $1 / 20$ event in the c50s05 ensemble), even when the inclination-semimajor axis correlation (Figure 15) is taken into account. However, if $\lambda$ is lower by $1 \sigma$ then the inclination of HD $17156 \mathrm{~b}$ lies in more plausible range $\left(p\left(I_{R}>37^{\circ}\right) \gtrsim 0.05\right.$ for the c10- ensembles, and $\sim 0.2$ for the c50s05 ensemble).

Collecting a larger sample of accurate measurements of the projected spin-orbit angle $\lambda$ for eccentric planets may prove to be a useful endeavor. The measurement of a significant misalignment in HD $17156 \mathrm{~b}$ suggests that misalignments are common; more comprehensive simulations of Stage 2 evolution can produce firm predictions for the dependence of the final inclination distribution on the initial conditions (initial number of planets, mass, semimajor axis, and inclination distribution, etc.); and other processes such as Kozai oscillations yield equally firm but quite different predictions.

\section{A MEASURE OF DYNAMICAL ACTIVITY}

The eccentricities of the three active ensembles with 10 initial planets, as well as the $e>0.2$ subsamples of all active ensembles, are pairwise consistent with being drawn from the same underlying distribution. The same holds true for the $e>0.2$ subsamples of all partially active ensembles. In $\$ 3$ we have taken this agreement as evidence that these ensembles have converged to the same eccentricity distribution. We hypothesize that this distribution, empirically described by a Schwarzschild distribution with $\sigma_{e} \sim 0.3$ (eq. 2), is the equilibrium endpoint of "dynamically active" planetary systems, where by "dynamically active" we mean systems whose planets experience strong mutual interactions and frequent encounters. We now attempt to find an empirical measure of whether a planetary system with given initial conditions will be dynamically active.

In the restricted three-body problem, the natural measure of the radius of influence of a planet on a nearby test particle in a nearly circular orbit is its Hill radius:

$$
R_{h}(r, M)=r\left[\frac{M}{3 M_{\odot}}\right]^{1 / 3}
$$

where $M$ and $r$ are the mass and orbital radius of the planet while $M_{\odot}$ is the mass of the star. In the case of two bodies with masses $M_{1}$ and $M_{2}$ that are small compared to $M_{\odot}$, on nearly circular orbits with similar radii $r$, the Hill radius is obtained from equation (3) by replacing $M$ with $M_{1}+M_{2}$ (Henon \& Petit 1986). For the purposes of this paper, where we must deal with planets having different masses and orbital radii, we define the mutual Hill radius

$$
R_{H}=\frac{1}{2}\left[R_{h}\left(r_{A}, M_{A}\right)+R_{h}\left(r_{B}, M_{B}\right)\right]
$$

as the average of the Hill radii of the two individual planets. This definition is somewhat arbitrary but reduces to the usual Hill radius when one planet is much more massive than the other.

In the case of the general three-body problem Marchal \& Bozis (1982) and Gladman (1993) have shown that two small planets on circular, nearly coplanar orbits can have no close encounters (are "Hill stable") if their semimajor axes are separated by ${ }^{14} a_{2}-a_{1}>$

14 Note that Gladman (1993) defines the mutual Hill radius as

$$
R_{H, G}=\frac{a_{1}+a_{2}}{2}\left(\frac{m_{1}+m_{2}}{3 M_{\odot}}\right)^{1 / 3}
$$




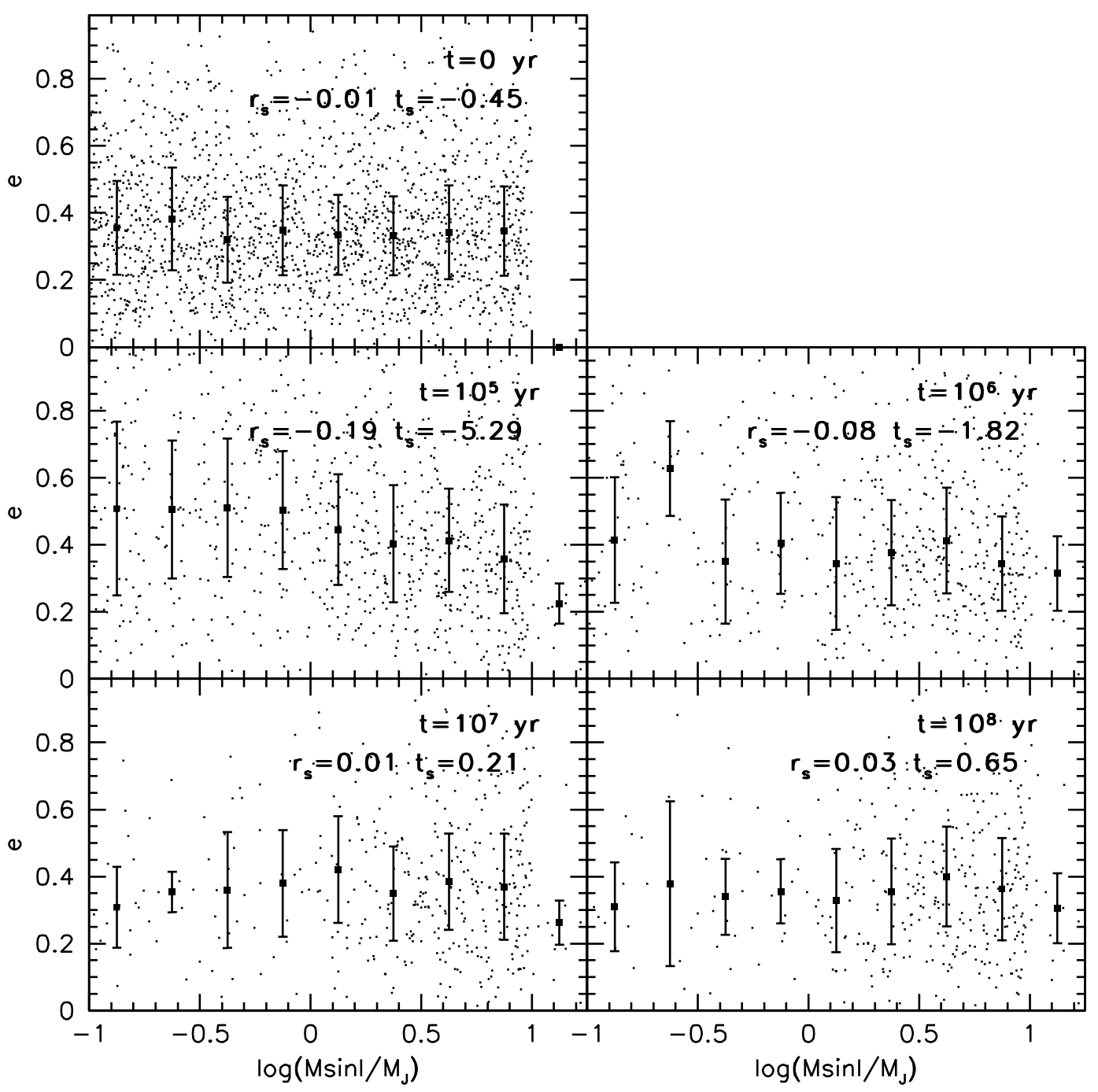

FIG. 11. - The evolution of the mass-eccentricity correlation in the c10s30 ensemble. The panels (left to right, top to bottom) show the masses and eccentricities of planets at times $t=0,10^{5}, 10^{6}, 10^{7}$, and $10^{8} \mathrm{yr}$. The meaning of the symbols is the same as in Figure 10

$2 \sqrt{3} R_{h}\left[\frac{1}{2}\left(a_{1}+a_{2}\right), M_{1}+M_{2}\right]$. For systems of three or more planets, this criterion still approximately predicts whether the system is unstable on short timescales. However, it does not accurately predict long-term stability (Chambers et al. 1996). Nevertheless, since it is usually the case that only the one or two closest neighbors (expressed in Hill radii) are responsible for most of the evolution, we can use the concept of Hill radii to explore approximate criteria for whether a given planetary system is dynamically active.

For a given planet $A$, we introduce the notion of its "Hill neighbor" $B$, which is the planet of larger mass whose orbit comes closest, in terms of mutual Hill radii, to the orbit of planet $A$. We define the Hill neighbor separation, $D_{H}$, to be the minimum distance between the orbital ellipses of planet $A$ and its Hill neighbor $B$, expressed in mutual Hill radii $R_{H}$. We use the minimum

In the limit of equal-mass planets on nearby circular orbits, definitions (4) and (5) differ by a factor of $\sqrt[3]{2}$. distance, instead of, say, the average distance, because the mutual interaction of the two planets is strongest at the point of closest approach.

For example, in a system with $N$ planets of different masses, there are $N-1$ Hill neighbors and $N-1$ Hill neighbor separations. In the n10s10 ensemble of Table 1 . there are 1000 systems of 10 planets each (at $t=0$ ), and thus 9000 Hill neighbors and 9000 Hill neighbor separations. We can observe the time evolution of this "Hill neighbor separation distribution" (NSD) and its statistical properties.

In Figure 16] we show the NSD of ensemble c10s40 at $t=0$ (top left panel) and its evolution from $t=10^{4} \mathrm{yr}$ (top right panel) through $t=10^{8} \mathrm{yr}$ (bottom right panel). At $t=0$ the systems of this ensemble are very tightly packed, and will be unstable by virtually any criterion based on Hill radii. The ensemble reacts to this strongly unstable situation by removing planets through collisions and ejections (see Figure 1) and by redistribut- 

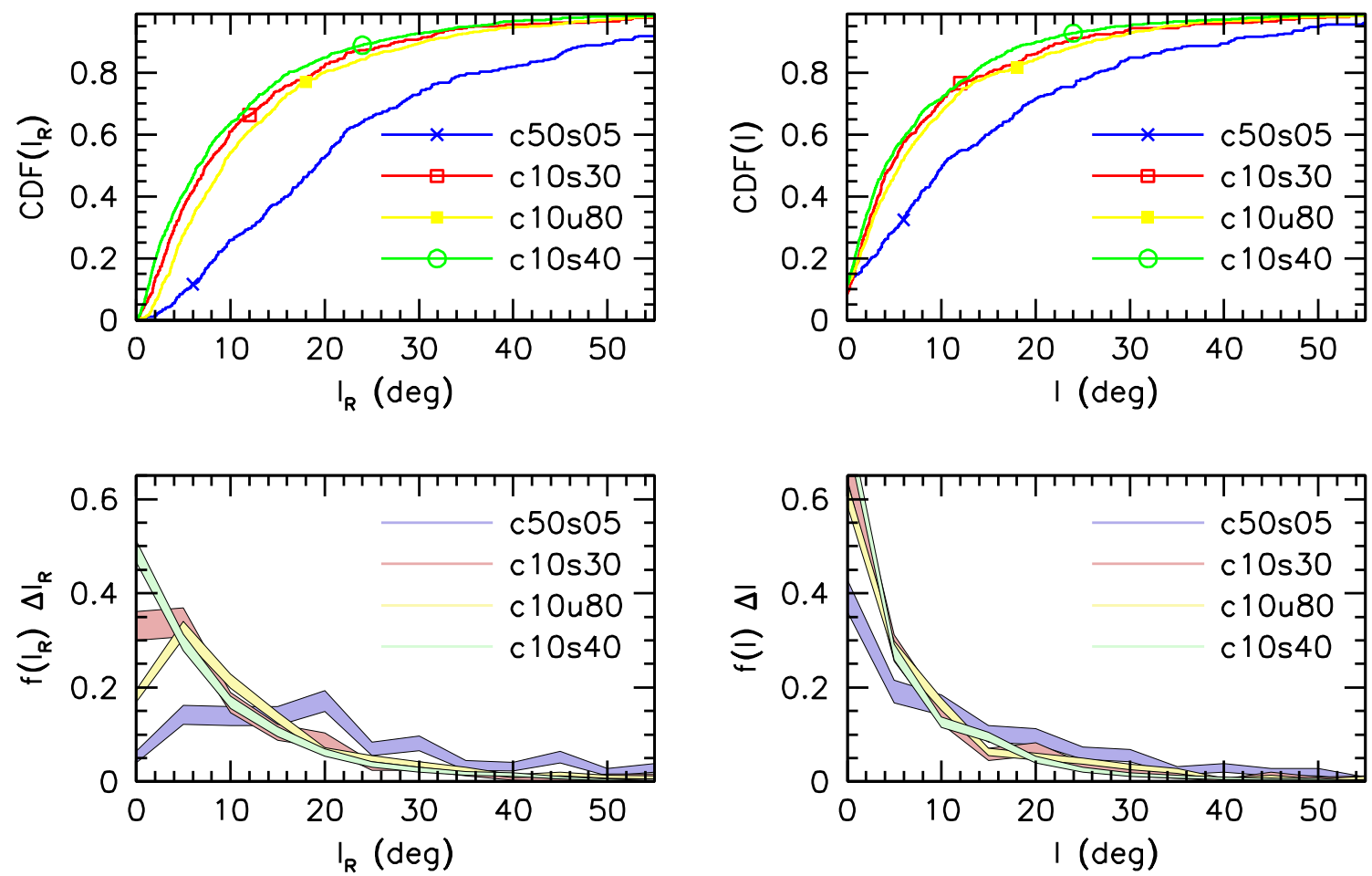

FIG. 12.- Cumulative (top row) and differential (bottom row) inclination distributions of active systems, calculated with respect to the reference plane of the initial conditions $\left(I_{R}\right.$, left panels) and the invariable plane at the end of the simulation ( $I$, right panels). The bin size of the differential distribution is $\Delta I_{R}=\Delta I=5^{\circ}$.

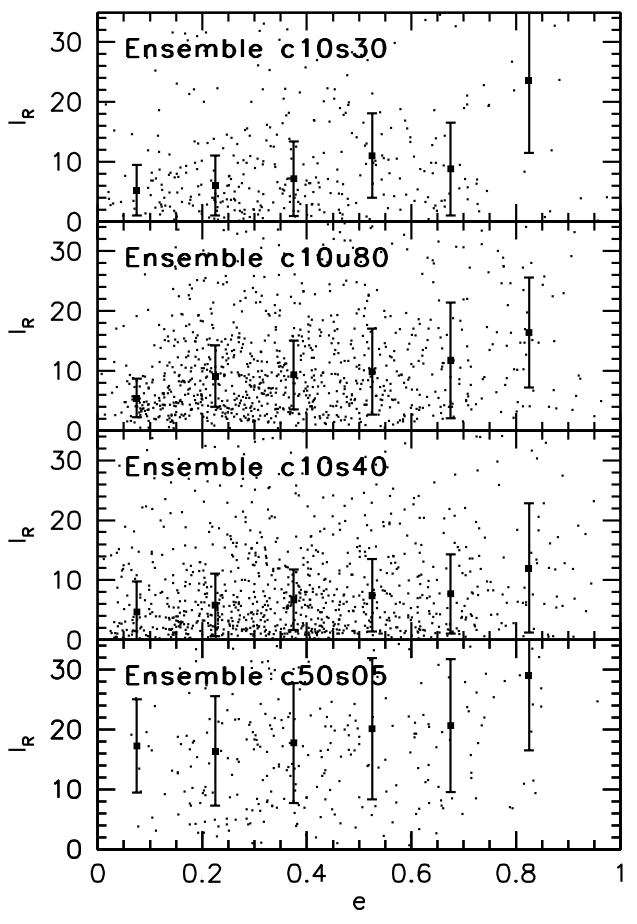

FIG. 13. - Correlation of inclination and eccentricity in active systems. The inclinations are with respect to the reference plane of the initial conditions.

ing planets so as to increase the spacing between their orbits. As a result, the number of planets with small $R_{H}$ decreases rapidly, and both the peak and the median of

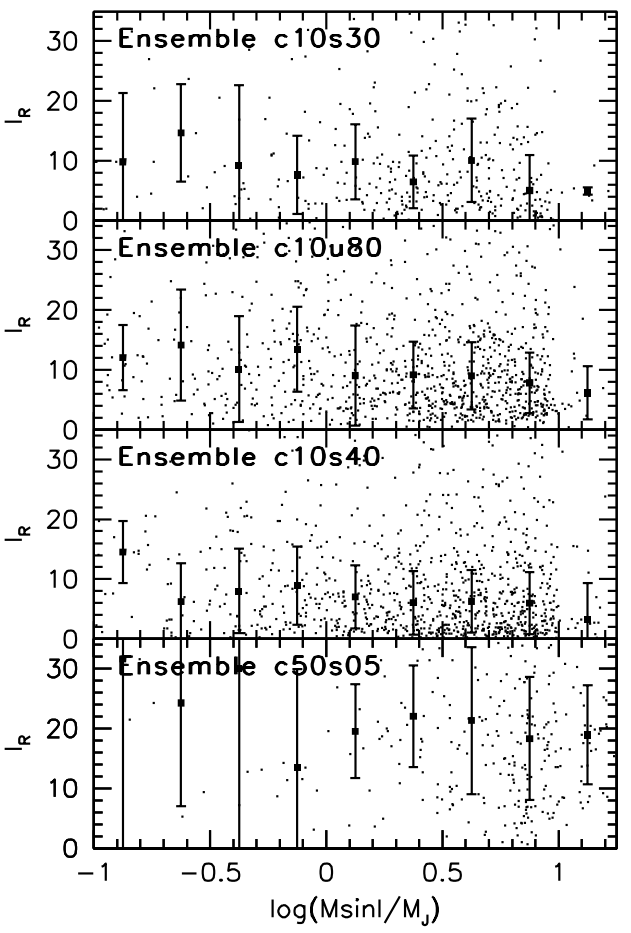

FIG. 14.- Correlation of inclination and $M \sin I$ in active systems. The inclinations are with respect to the reference plane of the initial conditions.

the NSD shift towards higher values of $R_{H}$. At $t=10^{8} \mathrm{yr}$ all neighbors are separated by more that $4 R_{H}$.

We repeat a similar analysis for all ensembles of Ta- 


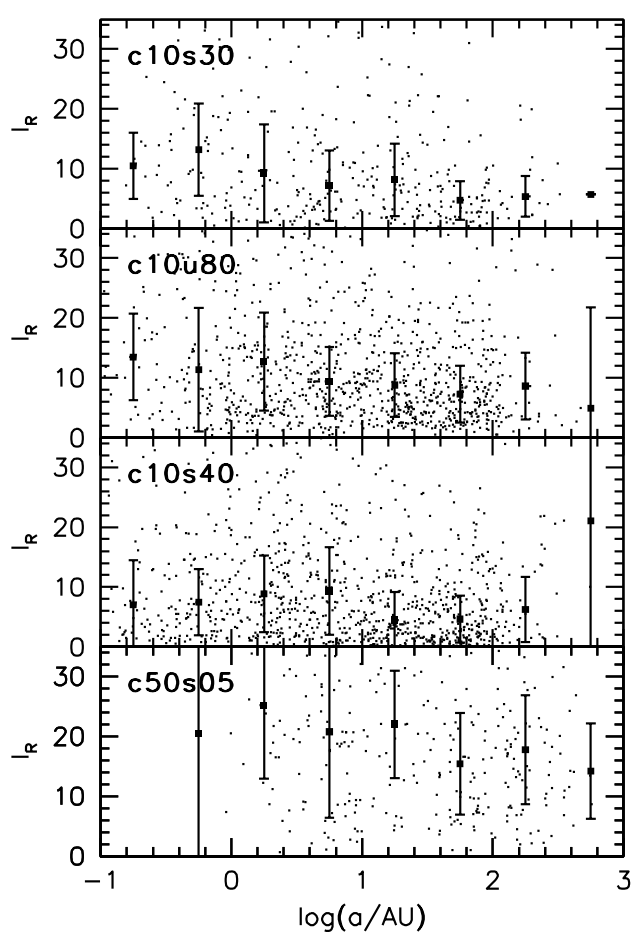

FIG. 15.- Correlation of inclination and semimajor axis in active systems. The inclinations are with respect to the reference plane of the initial conditions.

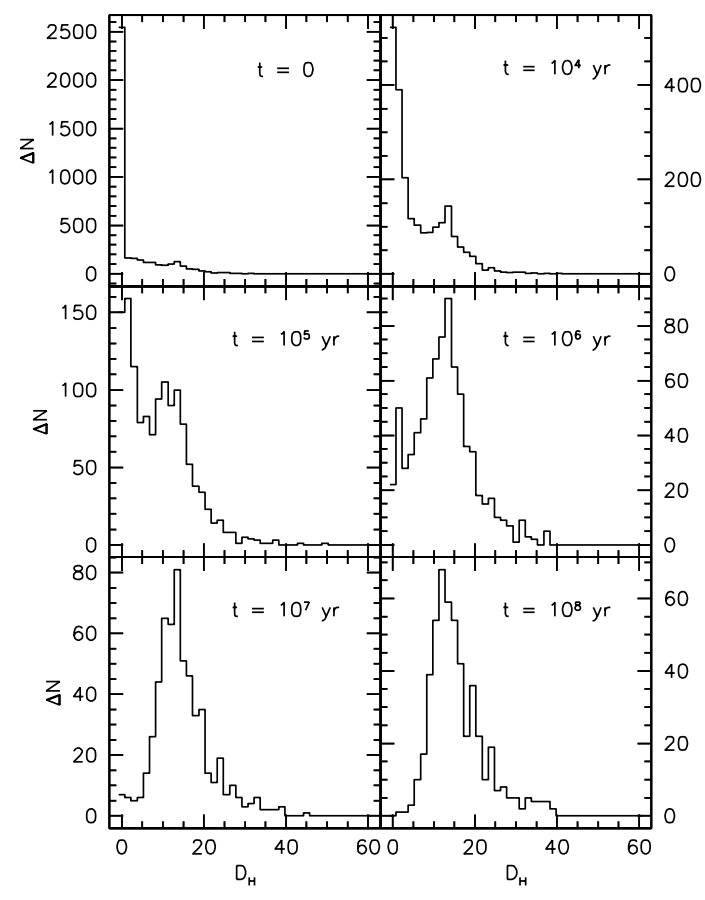

FIG. 16.- Evolution of the Hill neighbor separation distribution (NSD). The panels show (left to right, top to bottom) the evolution of the NSD of ensemble c10s40 at six different times. Plotted are the number of planets in the ensemble in bins of width $\Delta D_{H}=1.5$, where $D_{H}$ is the Hill neighbor separation (\$5).

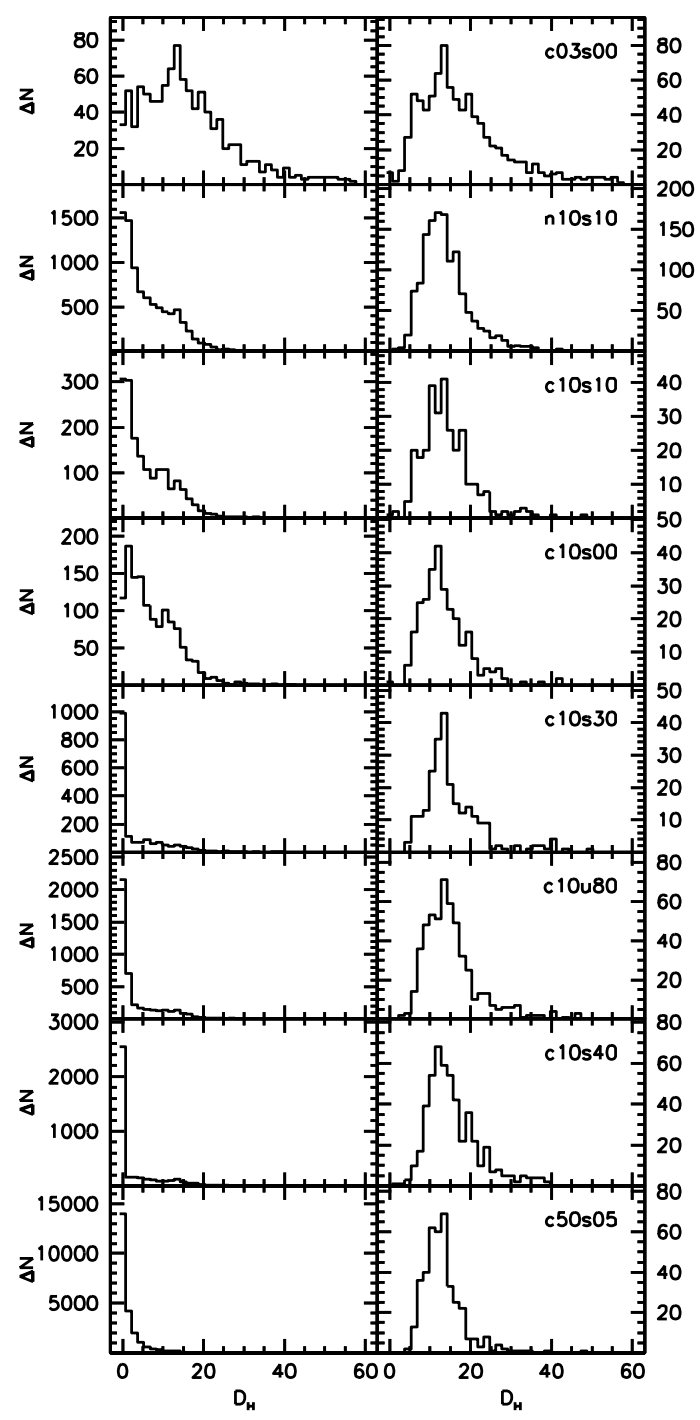

FIG. 17.- Comparison of initial and final Hill neighbor separation distributions (NSDs) of simulated ensembles. Each row shows the initial ( $t=0$, left panel) and final $\left(t=10^{8} \mathrm{yr}\right.$, right panel) NSD of the ensemble. Plotted are the number of planets in the ensemble in each bin of width $\Delta D_{H}=1.5$.

ble1. Figure 17] shows the initial (left column) and final (right column) NSD for each ensemble, starting with the one "inactive" ensemble in the top row, followed by three we classified as "partially active", and then the four "active" ensembles. A common property of all active ensembles is the strong peak at small values of $D_{H}$ in the initial distributions. For example, while all four active ensembles have initial median Hill neighbor separation $\widetilde{D}_{H}<1$, the lowest for a partially active ensemble (out of the three ensembles classified as such) is $\widetilde{D}_{H}=4.4$.

The final NSDs also share a number of common characteristics. All exhibit a sharp reduction in the number of objects at small values of $D_{H}$. This gap near $D_{H}=0$ is more pronounced in active ensembles. All examined NSDs peak at $D_{H} \simeq 12$, with the NSDs of active ensembles (bottom four panels) having a similar unimodal distribution with a strong peak at $D_{H} \simeq 12$, a width $\Delta D_{H} \sim 8(\mathrm{FWHM})$, and an extended tail reaching to 


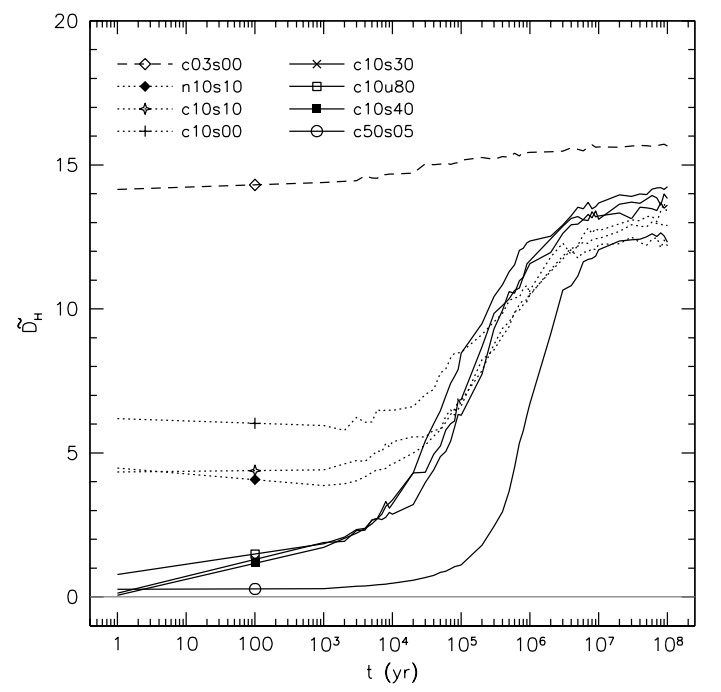

FIG. 18. - Evolution of median Hill neighbor separation $\widetilde{D}_{H}$ with time. The median is plotted against elapsed simulation time for each ensemble. Active, partially active, and inactive ensembles are plotted with solid, dotted and dashed lines, respectively.

much larger values of $D_{H}$.

Figure 18 shows the evolution of the median Hill neighbor separation $\widetilde{D}_{H}$ for the ensembles of Table 11. The division into dashed, dotted and solid ensembles, corresponding to inactive, partially active, and active ensembles, follows the convention adopted for Figure 3 and the separation into top, middle and bottom panels in Figure 2. The four ensembles with initial $\widetilde{D}_{H}<1$ are all active; the three having $4<\widetilde{D}_{H}<7$ are partially active, and the only ensemble with a large initial value $\widetilde{D}_{H} \simeq 14$ is inactive. All active and partially active ensembles converge to a final median Hill neighbor separation $\widetilde{D}_{H} \simeq 12-14$ after $10^{8}$ yr.

We take these results to offer hope that the initial value of $\widetilde{D}_{H}$ may be used as a crude measure of the dynamical activity of an ensemble, and hence as a predictor for the classification of the final eccentricity distribution. A much more thorough exploration of possible initial conditions is needed before we can tell whether this hope is justified. In the meantime, our best guess is that systems with $\widetilde{D}_{H} \lesssim 10$ are likely to be at least partially active.

Other statistical measures may eventually prove to be more useful or reliable in characterizing the dynamical activity of a planetary system. When devising the one employed here, we were guided by the criteria that it should reflect the level of short-term dynamical instability present in the system (satisfied by expressing the distances to the closest more massive neighbor in Hill radii), and that it should be applicable both to coplanar orbits and to orbits with significant inclinations (requiring the relatively complex definition of Hill neighbor separation). The statistic as defined above works reasonably well for systems of the type existing in our simulations - a few planets with a limited range of masses (two decades). However, care must be taken when applying it to (and interpreting it for) other types of systems, as we cannot prove that it will work everywhere equally well, and strongly suspect that there are possible, though per- haps pathological, planetary systems for which it does not work at all.

\section{DISCUSSION}

As described in the Introduction, the possibility that planet-planet interactions play a significant role in explaining the origin of the extrasolar planet eccentricity distribution has been discussed since soon after the first extrasolar planets were discovered. Most of these discussions focused either on exploring the dynamics of simplified two or three planet systems, or on following the dynamical evolution of planetary systems whose initial conditions were inspired by planetesimal accretion theory $^{15}$.

In this paper, we have integrated large ensembles of randomly constructed planetary systems over $100 \mathrm{Myr}$ timespans, simulating $P P \approx 5 \times 10^{12}$ planet-periods (eq. 11). The output from these simulations, and future simulations of this type, offers a rich resource for studies of Stage 2 planet evolution, and here we have focused on only a few aspects of this evolution, in particular the distributions of eccentricity, inclination, and separations. Initially, we classify the ensembles according to their final eccentricity distributions, and later show that this classification is strongly correlated with the initial median Hill neighbor separation $\widetilde{D}_{H}$, in that all ensembles classified as dynamically active had $\widetilde{D}_{H} \lesssim 1$ in the initial state. In all dynamically active ensembles that we have examined, we obtain the same final eccentricity distribution for a remarkably wide range of initial conditions. This distribution is described by a Schwarzschild distribution (eq. 2) with $\sigma_{e} \sim 0.3$. For $e \gtrsim 0.2$ the final eccentricity distribution in our simulations of active ensembles agrees with the observed eccentricity distribution of extrasolar planets remarkably well. The excess of observed systems with $e \lesssim 0.2$ may reflect either a population of planetary systems that are not dynamically active, or eccentricity damping by low-mass planets, planetesimals, or residual gas. In the former case, comparison with the observations suggests that about $25 \%$ of the known extrasolar systems with $P>20 \mathrm{~d}$ are inactive, and $75 \%$ active.

We find little or no correlation of other parameters (semimajor axis, planetary mass, and inclination) with the final eccentricity, although such correlations are present during periods of dynamical instability early on in the simulation.

This "equilibrium" distribution of eccentricity is a product of dynamical relaxation of an initially unstable system. The distribution is mostly established after $10^{7} \mathrm{yr}$ and remains stable to at least $10^{8} \mathrm{yr}$, where our simulations end. A few integrations have been carried to longer times, and show no evidence of further evolution. By $10^{8}$ yr most of the active ensembles have only $2-3$ remaining planets in the three decades of semimajor axis that we originally populated.

We find further that in all partially active and active ensembles that we examined, $\widetilde{D}_{H}$ converges to a common value between 12 and 14. In active ensembles, the distribution of $D_{H}$ converges to a common shape as well, with

15 In particular, the initial version of our paper was submitted to the arXiv preprint server on the same day as papers by Chatteriee et al. (2007) and Ford \& Rasio (2007), who reach many of the same conclusions. 
a peak at $D_{H} \simeq 12$ and a width $\Delta D_{H} \simeq 8$ (full width at half maximum). Thus both the eccentricity distribution and the distribution of Hill neighbor separations in active ensembles appear to be a common endpoint of the dynamical relaxation process.

An aggressive interpretation of the similarity of the observed and theoretical eccentricity distributions for $e \gtrsim 0.2$ is that the high eccentricities of observed planets have arisen as an endpoint of dynamical relaxation, by processes similar to those seen in the simulations of 3.1. long after the initial stage of planet formation and dispersal of the protoplanetary gas disk were complete. This interpretation leads to a number of interesting conclusions:

- There exists no single "right" ensemble of initial conditions at the end of Stage 1. Instead, there is a multitude of substantially different ensembles of initial conditions that lead to the same or similar final outcomes, at least for the eccentricity distribution and the distribution of Hill neighbor separations. An important corollary is that the details of initial conditions are impossible to deduce from the "relaxed" component of the observed eccentricity distribution, except to say that they are likely to be in the "active" regime where dynamical evolution is strong enough to drive the relaxation process.

- In a large fraction of systems the final products of Stage 1 planet formation must be dynamically active. This is in principle possible for both the planetesimal (e.g., Kokubo \& Ida 1998) and gravitational instability (e.g., Boss 2000) models. Of course, the separation into Stage 1 and Stage 2 is somewhat artificial, since the initial Stage 2 evolution occurs on timescales short compared to the likely duration of Stage 1.

- Planet-planet scattering in active ensembles changes the orbital planes of planets, broadening the distribution of inclinations with respect to the symmetry plane of the initial conditions. The same broadening is seen in simulations of three-planet scattering by Chatteriee et al. (2007). Assuming that the host-star spin vector is parallel to the symmetry axis of the initial protoplanetary disk, and that no other effects re-orient the spin axis of the star or the invariable plane of the planets (e.g., Tremaine 1991), this inclination distribution is detectable, at least in principle, in transiting planets through the Rossiter-McLaughlin effect. Given that the distribution of misalignments depends on the initial conditions more strongly than the distribution of eccentricities in active systems, its measurement in a large sample of extrasolar planets may yield valuable information about the endpoint of Stage 1 and the initial conditions for Stage 2 .

- The typical final number of giant planets in active systems is between 2 and 3, at least over the range of three decades in semimajor axis that we populate in the initial states (presumably other planets could form well outside this semimajor axis range, and their interactions with our simulated planets would be negligible). At $t=10^{8} \mathrm{yr}$, on average, $20 \%$ of active systems remain with only 1 planet, $75 \%$ have two or three planets, and only $5 \%$ have four or more planets, suggesting that most extrasolar planetary systems should have $2-3$ giant planets in this semimajor axis range (about one giant planet per decade). Consequently, extrasolar systems with a single detected eccentric planet are likely to harbor at least one more planet of comparable mass. Observational data show long-term radial velocity trends indicative of the presence of another planet in $\sim 50 \%$ of the currently known exoplanet systems (Butler et al. 2006). However, an equally interesting prediction is that such systems are also not likely to harbor more than one or two additional giant planets.

- These predictions are consistent with current observational data on the fraction of multi-planet systems. To compare our simulations directly to the observations, we cull them at age $10^{8} \mathrm{yr}$ to keep only those planets that produce a radial velocity semi-amplitude $K>10 \mathrm{~m} \mathrm{~s}^{-1}$ and have periods in the range $20 \mathrm{~d}<P<2500 \mathrm{~d}$ (the lower limit eliminates hot Jupiters, and the upper limit approximates the longest detectable orbital period). The culled ensembles c10s30, c10s40 and c10u80 predict a ratio of single- to multi-planet systems $\simeq 86: 14$ (84: 16 lowest, $89: 11$ highest), in excellent agreement with the $87: 13$ ratio seen when the observed sample subjected to same culling. The agreement is only weakly sensitive to the exact choice of threshold $K$ or the limits imposed on $P$. The prediction is less good for the ratio of semimajor axes of the outer $\left(a_{2}\right)$ and inner $\left(a_{1}\right)$ planet in multi-planet systems, where the simulations typically peak at $4<a_{2} / a_{1}<8$, while $80 \%$ of the observed ratios are in $1<a_{2} / a_{1}<4$ range. The distribution of semimajor axis ratios is not universal across the three ensembles either, pointing to a dependence on initial conditions that warrants further investigation. Finally, the c50s05 ensemble, due to the efficient clearing of the zone with $a<1$ AU (Figure 71), predicts no observed multi-planet systems at all.

- The typical final separation of planets in active multi-planet systems should be $\widetilde{D}_{H} \simeq 12-14$. The determination of this statistic in the currently known multiplanet systems is made difficult by the unknown inclinations, both the distribution of inclinations relative to the invariable plane and the inclination of the invariable plane to the line of sight. We define a new statistic $\widetilde{D}_{H}^{\prime}$, which is obtained from $\widetilde{D}_{H}$ by replacing all planet masses $M$ by $M \sin I$, and compute $\widetilde{D}_{H}^{\prime}$ for our ensembles by assuming that the normal to the invariable plane is distributed randomly and uniformly on a sphere and culling the ensembles using the same criteria on period and velocity amplitude described in the preceding paragraph. We obtain $\widetilde{D}_{H}^{\prime} \simeq 12$ 13. To compute the analogous statistic for the observations, we assume that the inclinations rel- 
ative to the invariable plane are described by a Schwarzschild distribution (Eq. 2) with $\sigma_{I}=10^{\circ}$, the distribution of the nodal longitudes $\Omega$ is uniform, and we take the longitudes of periastron $\varpi$ from Butler et al. (2006). We find $\widetilde{D}_{H}^{\prime} \simeq 8$ for the 13 known multi-planet systems. This agreement is probably adequate given the large statistical errors, although it is also possible that the observed systems can be stable at smaller values of $\widetilde{D}_{H}^{\prime}$ than our simulations because some of the planets are stabilized by mean-motion resonances.

- The median separation $\widetilde{D}_{H}$ in the solar system is 15.6 (four giant planets) or 21.2 (all eight planets). Our results therefore suggest that the solar system is inactive, which is consistent with the observation that the eccentricities of planets in the solar system are much lower than in extrasolar planetary systems ${ }^{16}$. Our crude estimate in 3.2 suggests that at least $25 \%$ of extrasolar planetary systems are inactive, so in this respect the solar system is not unusual.

- All active systems that we have examined eject a significant portion of the initial mass in planets. On average, the active systems we simulated ejected $50 \%$ of the initial mass $\left(10 M_{J}\right.$ ejected) if they started with 10 planets, and $80 \%\left(90 M_{J}\right.$ ejected) if they started with 50 . Therefore, free-floating planets should be common and have a number density roughly comparable to the number density of extrasolar planets. Such planets may be detected by future microlensing surveys, or in open clusters as planetary-mass objects not bound to stars (Zapatero Osorio et al. 2000).

The constraints and limitations of the above conclusions have to be kept in mind. The distribution of eccentricities is only a one-dimensional projection of the multidimensional distribution of orbital elements, masses and

16 Although the solar system appears to be inactive, in the sense that $\widetilde{D}_{H}$ appears to be larger than needed for long-term stability of the existing planets, there are almost no locations in the outer other properties, and it is likely that other statistics of this distribution do not approach universal values in active Stage 2 evolution (for example, the mass and semimajor axis distributions). It is also a priori possible that the equilibrium eccentricity distribution may be different for some ranges of initial conditions that we failed to explore in this paper. A much broader exploration of the possible initial conditions for Stage 2, and of the distribution of orbital elements and masses at the end of Stage 2 evolution, is needed to investigate these questions. It is particularly important to explore (i) a steeper initial mass function (more low-mass and fewer high-mass planets), since the final mass distributions in our simulations probably have too few low-mass planets (Figure 9); (ii) a minimum-mass cutoff lower than our current value of $0.1 M_{J}$, since a larger population of low-mass planets may damp eccentricities; (iii) tidal dissipation from the host star, which may affect orbits with pericenters less than a few stellar radii; (iv) active systems that initially contain giant planets only beyond a few $\mathrm{AU}$, to investigate what fraction of giant planets could acquire small semimajor axes through planet-planet interactions; (v) active systems containing terrestrial planets. Although investigating the wide range of possible initial conditions and final states of Stage 2 evolution is a massive task, the only major resource required for this investigation is processing time on cluster computers.

This research was supported in part by NASA grants NNG04H44g and NNX08AH83G, and used computational facilities supported by NSF grant AST-0216105. MJ gratefully acknowledges support from the Taplin Fellowship. The authors would like to thank Bruce T. Draine and Robert Lupton for helpful discussions of computational and statistical aspects of this work; Peter Goldreich, Fred Rasio and Vicky Kalogera for discussions; and the anonymous referee for thoughtful and detailed comments.

solar system (between Jupiter and Neptune) in which additional planets could be inserted on stable orbits (Holman 1997).

\section{REFERENCES}

Adams, F. C., \& Laughlin, G. 2003, Icarus, 163, 290

Bakos, G. Á., Kovács, G., Torres, G., Fischer, D. A., Latham, D. W., Noyes, R. W., Sasselov, D. D., Mazeh, T., Shporer, A., Butler, R. P., Stefanik, R. P., Fernández, J. M., Sozzetti, A., Pál, A., Johnson, J., Marcy, G. W., Winn, J. N., Sipőcz, B., Lázár, J., Papp, I., \& Sári, P. 2007, ApJ, 670, 826

Barbieri, M., Alonso, R., Laughlin, G., Almenara, J. M.,

Bissinger, R., Davies, D., Gasparri, D., Guido, E., Lopresti, C., Manzini, F., \& Sostero, G. 2007, A\&A, 476, L13

Binney, J., \& Tremaine, S. 2008, Galactic Dynamics, 2nd ed. (Princeton, NJ, Princeton University Press, 2008, 885 p.) Boss, A. P. 2000, ApJ, 536, L101

Butler, R. P., Wright, J. T., Marcy, G. W., Fischer, D. A., Vogt, S. S., Tinney, C. G., Jones, H. R. A., Carter, B. D., Johnson, J. A., McCarthy, C., \& Penny, A. J. 2006, ApJ, 646, 505 Chambers, J. E. 1999, MNRAS, 304, 793

Chambers, J. E., Wetherill, G. W., \& Boss, A. P. 1996, Icarus, 119,261

Chatterjee, S., Ford, E. B., \& Rasio, F. A. 2007, ArXiv

Astrophysics e-prints, astro-ph/0703166

Cumming, A. 2004, MNRAS, 354, 1165
Fabrycky, D., \& Tremaine, S. 2007, ApJ, 669, 1298

Fischer, D. A., Vogt, S. S., Marcy, G. W., Butler, R. P., Sato, B., Henry, G. W., Robinson, S., Laughlin, G., Ida, S., Toyota, E., Omiya, M., Driscoll, P., Takeda, G., Wright, J. T., \& Johnson, J. A. 2007, ApJ, 669, 1336

Ford, E. B., Havlickova, M., \& Rasio, F. A. 2001, Icarus, 150, 303

Ford, E. B., \& Rasio, F. A. 2007, ArXiv Astrophysics e-prints, astro-ph/0703163

Ford, E. B., Rasio, F. A., \& Yu, K. 2003, in Astronomical Society of the Pacific Conference Series, Vol. 294, Scientific Frontiers in Research on Extrasolar Planets, ed. D. Deming \& S. Seager, $181-188$

Gladman, B. 1993, Icarus, 106, 247

Goldreich, P., \& Sari, R. 2003, ApJ, 585, 1024

Henon, M., \& Petit, J.-M. 1986, Celestial Mechanics, 38, 67

Holman, M. J. 1997, Nature, 387, 785

Kokubo, E., \& Ida, S. 1998, Icarus, 131, 171

Levison, H. F., Lissauer, J. J., \& Duncan, M. J. 1998, AJ, 116 1998

Lin, D. N. C., \& Ida, S. 1997, ApJ, 477, 781 
Loeillet, B., Shporer, A., Bouchy, F., Pont, F., Mazeh, T., Beuzit, J. L., Boisse, I., Bonfils, X., Da Silva, R., Delfosse, X., Desort, M., Ecuvillon, A., Forveille, T., Galland, F., Gallenne, A., Hebrard, G., Lagrange, A. M., Lovis, C., Mayor, M., Moutou, C., Pepe, F., Perrier, C., Queloz, D., Segransan, D., Sivan, J. P., Santos, N. C., Tsodikovich, Y., Udry, S., \& Vidal-Madjar, A. 2007, ArXiv e-prints, ArXiv:0707.0679

Marchal, C., \& Bozis, G. 1982, Celestial Mechanics, 26, 311

Marcy, G., Butler, R. P., Fischer, D., Vogt, S., Wright, J. T., Tinney, C. G., \& Jones, H. R. A. 2005, Progress of Theoretical Physics Supplement, 158, 24

Marzari, F., \& Weidenschilling, S. J. 2002, Icarus, 156, 570

Naef, D., Latham, D. W., Mayor, M., Mazeh, T., Beuzit, J. L., Drukier, G. A., Perrier-Bellet, C., Queloz, D., Sivan, J. P., Torres, G., Udry, S., \& Zucker, S. 2001, A\&A, 375, L27

Nagasawa, M., Ida, S., \& Bessho, T. 2008, ApJ, 678, 498

Narita, N., Enya, K., Sato, B., Ohta, Y., Winn, J. N., Suto, Y., Taruya, A., Turner, E. L., Aoki, W., Yoshii, M., Yamada, T., \& Tamura, Y. 2007a, PASJ, 59, 763

Narita, N., Sato, B., Ohshima, O., \& Winn, J. N. 2007b, ArXiv e-prints, ArXiv:0712.2569

Navarro, J. F., Frenk, C. S., \& White, S. D. M. 1997, ApJ, 490, 493

Ohta, Y., Taruya, A., \& Suto, Y. 2005, ApJ, 622, 1118

Papaloizou, J. C. B., \& Terquem, C. 2001, MNRAS, 325, 221

Press, W. H., Teukolsky, S. A., Vetterling, W. T., \& Flannery, B. P. 1992, Numerical recipes in C. The art of scientific computing (Cambridge University Press, 2nd ed.)
Queloz, D., Eggenberger, A., Mayor, M., Perrier, C., Beuzit, J. L., Naef, D., Sivan, J. P., \& Udry, S. 2000, A\&A, 359, L13

Rasio, F. A., \& Ford, E. B. 1996, Science, 274, 954

Rauch, K. P., \& Holman, M. 1999, AJ, 117, 1087

Tabachnik, S., \& Tremaine, S. 2002, MNRAS, 335, 151

Tremaine, S. 1991, Icarus, 89, 85

Weidenschilling, S. J., \& Marzari, F. 1996, Nature, 384, 619

Winn, J. N., Johnson, J. A., Marcy, G. W., Butler, R. P., Vogt, S. S., Henry, G. W., Roussanova, A., Holman, M. J., Enya, K., Narita, N., Suto, Y., \& Turner, E. L. 2006, ApJ, 653, L69

Winn, J. N., Johnson, J. A., Peek, K. M. G., Marcy, G. W., Bakos, G. Á., Enya, K., Narita, N., Suto, Y., Turner, E. L., \& Vogt, S. S. 2007, ApJ, 665, L167

Winn, J. N., Noyes, R. W., Holman, M. J., Charbonneau, D., Ohta, Y., Taruya, A., Suto, Y., Narita, N., Turner, E. L., Johnson, J. A., Marcy, G. W., Butler, R. P., \& Vogt, S. S. 2005, ApJ, 631, 1215

Wisdom, J., \& Holman, M. 1991, AJ, 102, 1528

Wolf, A. S., Laughlin, G., Henry, G. W., Fischer, D. A., Marcy, G., Butler, P., \& Vogt, S. 2007, ApJ, 667, 549

Wu, Y., \& Murray, N. 2003, ApJ, 589, 605

Zapatero Osorio, M. R., Béjar, V. J. S., Martín, E. L., Rebolo, R., y Navascués, D. B., Bailer-Jones, C. A. L., \& Mundt, R. 2000, Science, 290, 103 